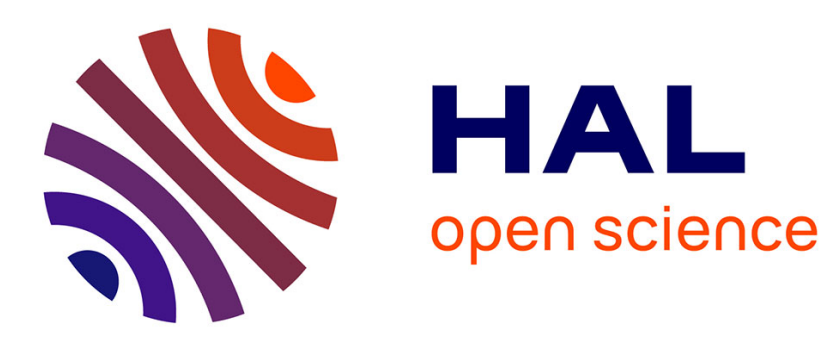

\title{
Trajectory Tracking For A Quadrotor Under Wind Perturbations: Sliding Mode Control With State-Dependent Gains
}

Gabriele Perozzi, Denis Efimov, Jean-Marc Biannic, Laurent Planckaert

\section{- To cite this version:}

Gabriele Perozzi, Denis Efimov, Jean-Marc Biannic, Laurent Planckaert. Trajectory Tracking For A Quadrotor Under Wind Perturbations: Sliding Mode Control With State-Dependent Gains. Journal of The Franklin Institute, inPress, 10.1016/j.jfranklin.2018.04.042 . hal-01798700

\section{HAL Id: hal-01798700 \\ https://inria.hal.science/hal-01798700}

Submitted on 7 Jun 2018

HAL is a multi-disciplinary open access archive for the deposit and dissemination of scientific research documents, whether they are published or not. The documents may come from teaching and research institutions in France or abroad, or from public or private research centers.
L'archive ouverte pluridisciplinaire HAL, est destinée au dépôt et à la diffusion de documents scientifiques de niveau recherche, publiés ou non, émanant des établissements d'enseignement et de recherche français ou étrangers, des laboratoires publics ou privés. 


\title{
Trajectory Tracking For A Quadrotor Under Wind Perturbations: Sliding Mode Control With State-Dependent Gains
}

Gabriele Perozzi $^{\mathrm{a}, *}$, Denis Efimov ${ }^{\mathrm{b}}$, Jean-Marc Biannic ${ }^{\mathrm{c}}$, Laurent Planckaert ${ }^{\mathrm{a}}$

${ }^{a}$ ONERA - The French Aerospace Lab, DAAA, 5 Bd Paul Painlevé BP 2126159014 Lille Cedex France.

${ }^{b}$ Inria, Non-A team, Parc Scientifique de la Haute Borne, 40 avenue Halley, Bât. A, Park Plaza, 59650 Villeneuve d'Ascq France.

${ }^{c}$ ONERA - The French Aerospace Lab, DTIS, BP74025 2 avenue Edouard Belin 31055

Toulouse Cedex 4 France.

\begin{abstract}
The problem of position tracking of a mini drone subject to wind perturbations is investigated. The solution is based on a detailed unmanned aerial vehicle (UAV) model, with aerodynamic coefficients and external disturbance components, which is introduced in order to better represent the impact of the wind field. Then, upper bounds of wind-induced disturbances are characterized, which allow a sliding mode control (SMC) technique to be applied with guaranteed convergence properties. The peculiarity of the considered case is that the disturbance upper bounds depend on the control amplitude itself (i.e. the system is nonlinear in control), which leads to a new procedure for the control tuning presented in the paper. The last part of the paper is dedicated to the analysis and reduction of chattering effects, as well as investigation of rotor dynamics issues. Conventional SMC with constant gains, proposed first order SMC, and proposed quasi-continuous SMC are compared. Nonlinear UAV simulator, validated through in-door experiments, is used to demonstrate the effectiveness of the proposed controls.
\end{abstract}

Keywords: Quadrotor modeling, sliding mode control, wind rejection,

\footnotetext{
*Corresponding author.

Email addresses: gabriele.perozzi@onera.fr (Gabriele Perozzi ), denis.efimov@inria.fr (Denis Efimov), jean-marc.biannic@onera.fr (Jean-Marc Biannic), laurent.planckaert@onera.fr (Laurent Planckaert)
} 
aerodynamic coefficients.

\section{Introduction}

In the last decades there has been a growing interest for small unmanned aerial vehicles both for military and commercial applications. They are useful in different domains such as monitoring, inspection, and other actions, especially in urban areas or nearby buildings and dangerous interiors, for natural calamities such as earthquakes. These machines are often required to move in unfamiliar environments in terms of geography and in terms of aerological conditions. In addition, the low mass of such units (comparing to the forces generated by the air disturbances) reduces significantly the domain of stable flight, then additional constraints have to be considered in the control design. Thus, it appears inevitable that, if we want to let UAVs operate in urban environments, inside turbulent air flow patterns for which accurate prediction is not possible a priori with limited resources, we need to focus on detailed aerodynamic models and sophisticated control laws. This paper is part of the four years project "Small drones in the wind" at ONERA Lille, which aims to use the drone itself as a wind sensor. First year was dedicated to the identification of the UAV model affected by wind velocity, which is used in this article and explained in [1]. This control design is carried out in parallel with development of a wind estimation tool in [? ].

The considered problem consists in design of a nonlinear robust control law, which ensures a stable and efficient navigation of a small UAV under unpredictable wind perturbations. The model described in [1] can also be used to estimate wind velocity, in this way the estimated values can themselves be used as inputs to the control to properly adapt the gains on-line and use this estimation in path planning and trajectory control to smartly avoid collisions.

There exist many control design techniques to counteract the effects of wind perturbations on flight of small UAVs, among which SMC plays a keyrole. Many methods have been proposed in the literature, for instance, some principal SMCs with their relative sliding surfaces and Lyapunov functions are illustrated in [2], [3]. Its insensitivity to the model errors, parametric uncertainties and other disturbances and its ability to globally stabilize the underactuated systems are two advantages of the sliding mode controller [4]. Sliding mode algorithms are extensively applied to dynamic systems and optimal algorithms are discussed in [5]. 
Dozens of articles have applied SMC methodology to UAVs in order to solve the position and the attitude tracking problems ensuring robustness against external disturbances. They have been compared extensively with respect to other controls in in-door experiments such as in [6], where SMC is compared with backstepping control for micro quadrotor. Chattering-free sliding mode controller is proposed in [7] by replacing a sign function with a high-slope saturation function, in [8] the energy saving effect because of chattering reduction is also evaluated. Second order sliding mode is used in [9] where two different sliding manifolds are defined for fully actuated and underactuated subsystems. In the paper [10] a robust backsteppingbased controller is proposed that induces integral sliding modes for the Newton-Euler underactuated dynamic model of a quadrotor subject to smooth bounded disturbances. The trajectory tracking of uncertain underactuated nonlinear dynamic systems is tackled by an adaptive fuzzy hierarchical slidingmode control in [11]. In the work [12] the controller of the fully actuated subsystem using a robust terminal sliding mode control algorithm is designed. A controller to provide robust position and attitude of the vehicle while relying only on knowledge of the limits of the disturbances is proposed in [13]. Sliding mode techniques are used also as observers estimators of the effect of the external disturbances such as wind and noise, and the whole observer-estimator-control law approach is presented in [14]. A SMC is proposed to stabilize a class of cascaded under-actuated systems, in which the UAV system is divided, in [15]. Famous super-twisting algorithm, which is able to ensure robustness with respect to bounded external disturbances, is discussed in [16]. In [17], the authors propose control algorithms similar to the ones described in this note, however they do not consider the rotors dynamics, the $3 \mathrm{D}$ trajectory, the first and second derivative of the UAV position and angles, making their approach interesting only in a mathematical point of view but impracticable in real 3D trajectory flight and far from the hover condition.

The quadrotor model itself is well established in the literature under various simplifications, in the present note we investigate the influence of the wind taking into account an identification work for the aerodynamic coefficients, which is useful for the control design. Two robust nonlinear SMC law design are described, which consider realistic assumptions on external disturbances of quadrotors. In the considered case the upper bound of matched disturbances depends nonlinearly on the control itself, the system state vector and wind disturbances. The closed-loop system stability is ensured for a 
selected maximum admissible value of the wind speed. The control strategy proposed in this article can be equipped with an additional wind estimator algorithm, as in [18], reducing automatically the control effort on the rotors when it is not necessary. However the peculiarity of the proposed SMC approach design is that the control allows the UAV to remain stable even without any coupled external disturbance observer.

The paper outline is as follows. In Section 2 the considered UAV drone is described and the flight dynamics model is derived. The control and disturbance bounds are calculated in Section 3. The two control designs are presented in Section 4, where the rotors issue, the chattering, the physical constraints and tools used for the implementation are also illustrated. The results of numerical experiments are shown in Section 5. The remarks and discussion conclude the paper in Section 6 .

\section{Quadrotor model}

The presented work is based on the commercial Parrot Ar. Drone 2.0 having configuration as it is shown in Fig. 1.

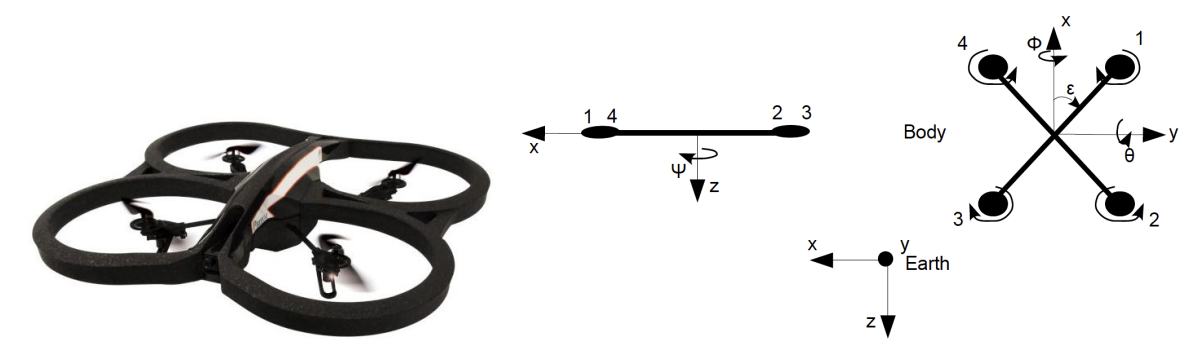

Figure 1: UAV Parrot Drone 2.0 and its configuration

This section presents a detailed model of the UAV dynamics used to develop the controller and to estimate the wind velocity, making the drone as a wind sensor.

\subsection{Quadrotor flight dynamics}

According to the identification work for the Parrot AR Drone 2.0 in [1] performed at maximal speeds in forward, lateral, and vertical directions respectively $\pm 5, \pm 5 \pm 1 \mathrm{~m} / \mathrm{s}$ (as in Table 3 ), rotor gyroscopic effects and 
inertial counter torques can be neglected since they are rather small. Then the translation dynamics of the drone in the body frame yield

$$
m\left[\begin{array}{c}
\dot{u} \\
\dot{v} \\
\dot{w}
\end{array}\right]+m\left[\begin{array}{c}
p \\
q \\
r
\end{array}\right] \times\left[\begin{array}{c}
u \\
v \\
w
\end{array}\right]=\left[\begin{array}{c}
F_{\text {Xaero }} \\
F_{\text {Yaero }} \\
F_{\text {Zaero }}
\end{array}\right]+m R^{T}\left[\begin{array}{l}
0 \\
0 \\
g
\end{array}\right]
$$

which can be represented in the earth frame

$$
m\left[\begin{array}{c}
\ddot{x} \\
\ddot{y} \\
\ddot{z}
\end{array}\right]=R\left[\begin{array}{c}
F_{\text {Xaero }} \\
F_{\text {Yaero }} \\
F_{\text {Zaero }}
\end{array}\right]+m\left[\begin{array}{l}
0 \\
0 \\
g
\end{array}\right]
$$

where $m$ is the mass of the UAV, $u, v, w$ are its linear velocities expressed in body frame, $p, q, r$ are its angular velocities in body frame, $F_{X a e r o}, F_{\text {Yaero }}, F_{\text {Zaero }}$ are the external aerodynamic forces in body frame, $g$ is is the gravity acceleration in inertial frame, $R$ is the rotational matrix defined as

$$
R=\left[\begin{array}{ccc}
c_{\psi} c_{\theta} & -s_{\psi} c_{\phi}+c_{\psi} s_{\theta} s_{\phi} & s_{\phi} s_{\psi}+c_{\psi} s_{\theta} c_{\phi} \\
s_{\psi} c_{\theta} & c_{\psi} c_{\phi}+s_{\psi} s_{\theta} s_{\phi} & -c_{\psi} s_{\phi}+s_{\psi} s_{\theta} c_{\phi} \\
-s_{\theta} & c_{\theta} s_{\phi} & c_{\theta} c_{\phi}
\end{array}\right]
$$

where $c_{\psi}=\cos (\psi), s_{\psi}=\sin (\psi)$ and similar for the other angles. Then the passage from the earth frame $\left(\mathcal{R}_{0}\right)$ to the body one $(\mathcal{R})$ is

$$
\left[X^{T}\right]_{\mathcal{R}}=\left[X^{T}\right]_{\mathcal{R}_{0}} R
$$

The rotational dynamics of the drone with respect to inertial earth frame are

$$
I\left[\begin{array}{c}
\dot{p} \\
\dot{q} \\
\dot{r}
\end{array}\right]=-\left[\begin{array}{c}
p \\
q \\
r
\end{array}\right] \times I\left[\begin{array}{c}
p \\
q \\
r
\end{array}\right]+\left[\begin{array}{c}
L_{\text {aero }} \\
M_{\text {aero }} \\
N_{\text {aero }}
\end{array}\right]
$$

with the inertia matrix

$$
I=\left[\begin{array}{ccc}
I_{x x} & 0 & 0 \\
0 & I_{y y} & 0 \\
0 & 0 & I_{z z}
\end{array}\right]
$$

where $L_{\text {aero }}, M_{\text {aero }}, N_{\text {aero }}$ are the external aerodynamic moments in the body frame. 
The relation between angular velocities and Euler angles

$\dot{\phi}=p+\tan \theta(q \sin \phi+r \cos \phi), \quad \dot{\theta}=q \cos \phi-r \sin \phi, \quad \dot{\psi}=\frac{q \sin \phi+r \cos \phi}{\cos \theta}$,

are considered avoiding the singularity at $\theta=\frac{\pi}{2}$, which is a reasonable assumption in our case since the topic of this article is not to achieve extreme maneuvers.

Hence the full model of the system is presented by the equations (2), (4), $(5)$. The aerodynamic forces $F_{\text {Xaero }}, F_{Y a e r o}, F_{Z a e r o}$, moments $L_{\text {aero }}, M_{\text {aero }}, N_{\text {aero }}$, and related coefficients are derived below using a combination of momentum and blade element theory in helicopters, well explained in [19], [20], [21].

\subsection{Quadrotor aerodynamics}

Aerodynamic forces and moments for each rotor, where subscript $j$ indicates the $j^{\text {th }}$ rotor, are derived as

$$
\begin{aligned}
F_{X_{j}} & =-\rho A R^{2} \frac{u_{j}-u_{w}}{\sqrt{\left(u_{j}-u_{w}\right)^{2}+\left(v_{j}-v_{w}\right)^{2}}} C_{H j} \omega_{j}^{2}, \\
F_{Y_{j}} & =-\rho A R^{2} \frac{v_{j}-v_{w}}{\sqrt{\left(u_{j}-u_{w}\right)^{2}+\left(v_{j}-v_{w}\right)^{2}}} C_{H j} \omega_{j}^{2}, \\
F_{Z_{j}} & =-\rho A R^{2} C_{T_{j}} \omega_{j}^{2} \\
L_{j} & =-\operatorname{sign} \omega_{j} \rho A R^{3} \frac{u_{j}-u_{w}}{\sqrt{\left(u_{j}-u_{w}\right)^{2}+\left(v_{j}-v_{w}\right)^{2}}} C_{R m j} \omega_{j}^{2}, \\
M_{j} & =-\operatorname{sign} \omega_{j} \rho A R^{3} \frac{v_{j}-v_{w}}{\sqrt{\left(u_{j}-u_{w}\right)^{2}+\left(v_{j}-v_{w}\right)^{2}}} C_{R m j} \omega_{j}^{2}, \\
N_{j} & =-\operatorname{sign} \omega_{j} \rho A R^{3} C_{Q_{j}} \omega_{j}^{2},
\end{aligned}
$$

where $\rho$ is the air density, $A$ is the rotor area, $R$ is the rotor radius, $\left(u_{w}, v_{w}, w_{w}\right)$ are the wind velocities with respect to the earth in body frame respectively in $(x, y, z)$ directions, $C_{H j}$ is the hub force coefficient, $C_{T j}$ is the rotor thrust coefficient, $\omega_{j}$ is the rotor angular speed, $C_{Q_{j}}$ is the rotor drag moment coefficient, $C_{R m j}$ is the rotor rolling moment coefficient.

Total aerodynamic forces are

$$
F_{\text {Xaero }}=\sum_{j=1}^{4} F_{X j}, \quad F_{\text {Yaero }}=\sum_{j=1}^{4} F_{Y j}, \quad F_{\text {Zaero }}=\sum_{j=1}^{4} F_{Z j}
$$


Total aerodynamic moments are

$$
\begin{gathered}
L_{\text {aero }}=\sum_{j=1}^{4}\left(L_{j}+F_{Z j} l \mathrm{~s}_{j}-h F_{Y j}\right), \quad M_{\text {aero }}=\sum_{j=1}^{4}\left(M_{j}-F_{Z j} l \mathrm{c}_{j}+h F_{X j}\right), \\
N_{\text {aero }}=\sum_{j=1}^{4}\left(N_{j}+F_{Y j} l \mathrm{c}_{j}-F_{X j} l \mathrm{~s}_{j}\right),
\end{gathered}
$$

where $h$ is the distance between rotors plane and the center of gravity of the $\mathrm{UAV}, l$ is the arm length, and with

$$
\mathrm{c}_{j}=\cos \left(\frac{\pi}{2}(j-1)+\epsilon\right), \quad \mathrm{s}_{j}=\sin \left(\frac{\pi}{2}(j-1)+\epsilon\right),
$$

where in our UAV configuration we have $\epsilon=\frac{\pi}{4}$. Thus, for vectors $\mathrm{c}_{j}$ and $\mathrm{s}_{j}$ we have cosines and sinus of the angles $\left[\frac{\pi}{4}, \frac{3}{4} \pi, \frac{5}{4} \pi, \frac{7}{4} \pi\right]$. The blade flapping can be modeled as a fictitious hinge spring.

The aerodynamic coefficients $\left(C_{T}\right.$ coefficient of the thrust, $C_{R m}$ coefficient of the rolling moment, $C_{H}$ coefficient of the hub force, $C_{Q}$ coefficient of the 
drag moment, $\mu$ advance ratio, $\lambda$ inflow ratio) are defined as follows

$$
\begin{aligned}
& C_{R m j}=\sigma a\left(\frac{\mu_{j}}{8}\left(\lambda_{j}-\frac{4}{3} \theta_{0}+\theta_{t w}\right)+\frac{b_{1}}{16}\left(1-\frac{\mu_{j}^{2}}{2}\right)\right), \\
& C_{T j}=\sigma a\left(\left(1+\frac{3}{2} \mu_{j}^{2}\right) \frac{\theta_{0}}{6}-\left(1+\mu_{j}^{2}\right) \frac{\theta_{t w}}{8}-\frac{\lambda_{j}}{4}\right), \\
& \lambda_{j}=\sigma a \frac{\left(1+\frac{3}{2} \mu_{j}^{2}\right) \frac{\theta_{0}}{6}-\left(1+\mu_{j}^{2}\right) \frac{\theta_{t w}}{8}-\frac{\lambda_{j}}{4}}{2 \sqrt{\mu_{j}^{2}+\lambda_{j}^{2}}}+\frac{w_{w}-w_{j}+q l \mathrm{c}_{j}-p l \mathrm{~s}_{j}}{R\left|\omega_{j}\right|}, \\
& \mu_{j}=\frac{1}{R\left|\omega_{j}\right|} \sqrt{\left(u_{j}-u_{w}\right)^{2}+\left(v_{j}-v_{w}\right)^{2}}, \quad C_{Q_{j}}=C_{Q_{P j}}+C_{Q_{i j}}, \\
& \frac{C_{Q_{P j}}}{\sigma}=\frac{1}{8}\left(C_{D 0}+C_{D i} \theta_{0}^{2}\right)\left(1+\mu_{j}^{2}\right)-C_{D i} \theta_{0} \theta_{t w}\left(\frac{1}{5}+\frac{\mu_{j}^{2}}{6}\right)+C_{D i} \theta_{t w}^{2}\left(\frac{1}{12}+\frac{\mu_{j}^{2}}{16}\right) \\
& -C_{D i} \lambda_{j}\left(\frac{\theta_{0}}{3}-\frac{\theta_{t w}}{4}\right)+C_{D i}\left(\frac{\mu_{j}^{2}}{8}\left(a_{0}^{2}+\frac{a_{1}^{2}}{4}+\frac{3 b_{1}^{2}}{4}\right)+\frac{1}{16}\left(a_{1}^{2}+b_{1}^{2}\right)+\frac{\lambda_{j}^{2}}{4}\right. \\
& \left.+\frac{\mu_{j}}{6} a_{0} a_{1}-\frac{\lambda_{j} \mu_{j}}{4} b_{1}\right) \\
& \frac{C_{Q_{i j}}}{\sigma a}=\lambda_{j}\left(\frac{\theta_{0}}{6}-\frac{\theta_{t w}}{8}-\frac{\lambda_{j}}{4}\right)-\frac{\mu_{j}^{2}}{8}\left(a_{0}^{2}+\frac{a_{1}^{2}}{4}+\frac{3 b_{1}^{2}}{4}\right)-\frac{1}{16}\left(a_{1}^{2}+b_{1}^{2}\right)-\frac{\mu_{j}}{6} a_{0} a_{1} \\
& +\frac{\lambda_{j} \mu_{j}}{4} b_{1}, \quad C_{H j}=C_{H P_{j}}+C_{H i j}, \\
& \frac{C_{H P j}}{\sigma}=\frac{\mu_{j}}{4}\left(C_{D 0}+C_{D i} \theta_{0}^{2}\right)+C_{D i}\left(\frac{\mu_{j}}{24}\left(3 \theta_{t w}^{2}-8 \theta_{0} \theta_{t w}\right)+\frac{\theta_{0}}{24}\left(3 \mu_{j}^{2} b_{1}-12 \lambda_{j} \mu_{j}\right.\right. \\
& \left.\left.-4 b_{1}\right)-\frac{\theta_{t w}}{16}\left(\mu_{j}^{2} b_{1}-4 \lambda_{j} \mu_{j}-2 b_{1}\right)+\frac{\mu_{j}^{2}}{8} a_{0} a_{1}+\frac{\mu_{j}}{16}\left(a_{1}^{2}-b_{1}^{2}\right)+\frac{1}{4} \lambda_{j} b_{1}\right), \\
& \frac{C_{H i j}}{\sigma a}=\frac{\theta_{0}}{4}\left(\lambda_{j} \mu_{j}+\frac{2}{3} b_{1}\right)-\frac{\theta_{t w}}{8}\left(\lambda_{j} \mu_{j}+b_{1}\right)+\frac{\mu_{j}}{8}\left(a_{0}^{2}+b_{1}^{2}\right)-\frac{3}{8} \lambda_{j} b_{1}+\frac{1}{12} a_{0} a_{1},
\end{aligned}
$$

where $w_{w}$ is the wind velocity with respect to the earth in body frame in $z$ direction, $\sigma$ is the rotor solidity ratio, $a$ is the lift curve slope of the blade section, $C_{D 0}$ is the drag coefficient of the blade section, $C_{D i}$ is the induced drag coefficient of the blade section, $\theta_{0}$ is the angle of attack of the root profile, $a_{0}, a_{1}, b_{1}$ are the coefficients of the blade flapping equation, $\lambda$ is the inflow ratio, $\mu$ is the advance ratio, subscript stat indicates the value in stationary phase. The shape of above UAV coefficients can be explained recalling aerodynamic science: the thrust is the resultant of the vertical forces acting on all the blade elements. The inflow ratio is the ratio between the 
component of UAV velocity perpendicular to the rotor disk with respect to the blade tip speed. The advance ratio indicates the component of the UAV velocity parallel to the rotor disk with respect to the blade tip speed. The rolling moment of a propeller exists in forward flight when the advancing blade is producing more lift than the retreating one and it is the integration over the entire rotor of the lift of each section acting at a given radius. The hub force is the resultant of the horizontal forces acting on all the blade elements. The drag moment about the rotor shaft is caused by the aerodynamic forces acting on the blade elements, the horizontal forces acting on the rotor are multiplied by the moment arm and integrated over the rotor.

Identification results thanks to indoor experiments on Parrot drone at low/moderate velocity, validates the previous UAV model and allows some simplifications to be accepted: $\theta_{t w}=0 . \quad C_{D i}=0 . \quad \lambda_{j}=\lambda_{\text {stat }}-$ $\frac{4}{\sigma a} K_{z} \frac{w_{j}-w_{w}}{R\left|\omega_{j}\right|}, K_{z}, \lambda_{\text {stat }} \geq 0$, where $K_{z}$ (as in Table 1 and by [1]) comes from the approximation of the $\lambda$ equation in vertical flight. $C_{T j}=C_{T \text { stat }}+$ $K_{z} \frac{w_{j}-w_{w}}{R\left|\omega_{j}\right|}, C_{T s t a t} \geq 0$, such models of $\lambda_{j}$ and $C_{T j}$ are rather precise in the climbing phase, but less accurate in descent phase, since the model tends to slightly overestimate the propulsion in the descent phase. $C_{H j}=K_{D} \mu_{j}, K_{D} \geq$ 0 , the UAV drag is modeled as $\rho A R^{2} \sum C_{H j} \omega_{j}^{2}$, corresponding to the rotors at low UAV speed, otherwise at higher speed we need to add the body drag effect since it depends on square of velocity. However the constant $K_{D}$, (as in Table 1 and by [1]) has been identified for the forward velocity less than $5 \mathrm{~m} / \mathrm{s}$ taking into account the interactions between the rear and the front rotors, and considering the whole UAV body and rotors.

\subsection{Rotors dynamics}

The rotors are driven by DC-motors, which binds electrical and mechanical quantities. As in [6] [22], rotors can be represented as in Fig. 2, and described by the dynamic system

$$
\begin{cases}L \frac{d i}{d t} & =u-R i-K_{e} \omega_{m} \\ J \frac{d \omega_{m}}{d t} & =\tau_{m}-\tau_{d}\end{cases}
$$

where $u$ is the motor input, $R$ is the motor inertial resistance, $L$ is the motor inductance, $K_{e}$ is the motor constant corresponding to the back EMF constant, $\tau_{m}$ is the motor torque, $\omega_{m}$ is the motor angular speed, $\tau_{d}$ is the motor load, $J$ is the motor moment of inertia, $i$ is the current. Considering a small motor with a very low inductance the given system can be simplified and 


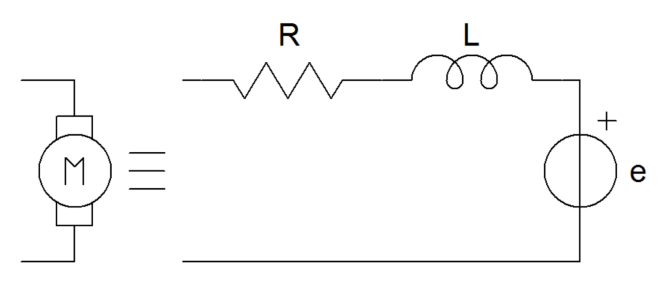

Figure 2: Rotor model

approximated, and linearizing around an operating point $\omega_{0}$, the system takes the form of $\dot{\omega}_{m}=-A \omega_{m}+B u+C$, where $A, B, C$ are rotors parameters, which can be further identified as:

$$
G(s)=\frac{1}{b s+1},
$$

where the time constant $b=0.1$ can be found considering Parrot Drones. Time constant slightly varies based on the considered motor and thus on the considered UAV mass. Precisely, in the Parrot Drones, the time constant is less than 0.1 for the increasing rotor angular velocities, and it is almost equal to 0.1 for decreasing rotor angular velocities.

In this section, two UAV models with different complexity have been presented (a complete one, and a reduced model for low/moderate velocity). The model with simplifications, which are accepted following [1], will be used to design a SMC, the other one will be used to validate the control in numerical experiments, since it considers nonlinear coefficients and a more realistic UAV behavior.

\section{Control system equations}

The model presented in previous section can be rewritten in the state-space form

$$
\dot{X}=f(X, U, d)
$$

where $f$ is expressed in (1), (4), (5) and the state vector $X$ is chosen as

$$
X=\left[\begin{array}{llllllllllll}
x & y & z & \dot{x} & \dot{y} & \dot{z} & \phi & \theta & \psi & p & q & r
\end{array}\right]^{T},
$$

$U=\left[\begin{array}{llll}U_{z} & U_{\theta} & U_{\phi} & U_{\psi}\end{array}\right]^{T}$ is the control input, and disturbances $d$ are described in the next subsection. The relations between the control inputs and the rotor 
velocities are defined by an invertible matrix

$$
\left[\begin{array}{c}
U_{z} \\
U_{\theta} \\
U_{\phi} \\
U_{\psi}
\end{array}\right] \approx\left[\begin{array}{cccc}
K_{f} & K_{f} & K_{f} & K_{f} \\
K_{f} l \mathrm{c}_{j} & K_{f} l \mathrm{c}_{j} & K_{f} l \mathrm{c}_{j} & K_{f} l \mathrm{c}_{j} \\
-K_{f} l \mathrm{~s}_{j} & -K_{f} l \mathrm{~s}_{j} & -K_{f} l \mathrm{~s}_{j} & -K_{f} l \mathrm{~s}_{j} \\
K_{m} & -K_{m} & K_{m} & -K_{m}
\end{array}\right]\left[\begin{array}{c}
\omega_{1}^{2} \\
\omega_{2}^{2} \\
\omega_{3}^{2} \\
\omega_{4}^{2}
\end{array}\right]
$$

with $\omega_{\min } \leq \omega_{j} \leq \omega_{\max }$, where $K_{f}=\rho A R^{2} C_{T \text { stat }}$ and $K_{m}=\rho A R^{3}\left(\frac{\sigma C_{D 0}}{8}+\right.$ $\left.\lambda_{\text {stat }} \sigma a\left(\frac{\theta_{0}}{6}-\frac{\lambda_{\text {stat }}}{4}\right)\right)$. In this work, the control inputs are selected to be proportional to the terms with $\omega_{j}^{2}$. Thus, expanding (1) and (4), the other terms dependent linearly on $\omega_{j}$ and wind velocities are considered as disturbances. Since we do not know in advance the wind perturbations, then we cannot use these terms in controls. Such a decomposition of thrust (which is proportional to $\omega_{j}$ and $\omega_{j}^{2}$ ) and selection of disturbances are almost exact in the hover flight, where we have $(p, q, r) \approx(\dot{\phi}, \dot{\theta}, \dot{\psi})$.

In the following, linear rotors speed in body frame are computed as a function of the state in body frame

$$
\left[\begin{array}{c}
u_{j} \\
v_{j} \\
w_{j}
\end{array}\right]=\left[\begin{array}{l}
p \\
q \\
r
\end{array}\right] \times\left[\begin{array}{c}
l c_{j} \\
l s_{j} \\
h
\end{array}\right]+\left[\begin{array}{c}
u \\
v \\
w
\end{array}\right] .
$$

\subsection{Definition of disturbances}

In the literature, it is often assumed that each component of the disturbance input vector $d$ admits a fixed upper bound, which means $|d| \leq D$ for some known $D \geq 0$. Unfortunately, it is a rather conservative hypothesis, and that is why the varying state-dependent bounds will be considered in our case for $d$. However, we will assume boundedness of the wind velocities: $\left|u_{w}\right| \leq D_{x},\left|v_{w}\right| \leq D_{y},\left|w_{w}\right| \leq D_{z}$, for some known $D_{x} \geq 0, D_{y} \geq 0, D_{z} \geq 0$, which is a reasonable restriction.

In this work we will call signals, whose influence is compensated by robust abilities of the control, as "disturbances", which include external inputs (the wind velocities), state components and controls. Substituting (8), (6) and (7) in (1), (4), and performing calculations with the indicated simplifications, we have the disturbance $d=\left[d_{x}, d_{y}, d_{z}, d_{\phi}, d_{\theta}, d_{\psi}\right]$ represented by the following 
terms

$$
\begin{aligned}
d_{x} & =\sum_{j=1}^{4}-\rho A R K_{D}\left(u_{j}-u_{w}\right)\left|\omega_{j}\right| \\
d_{y} & =\sum_{j=1}^{4}-\rho A R K_{D}\left(v_{j}-v_{w}\right)\left|\omega_{j}\right| \\
d_{z} & =\sum_{j=1}^{4}-\rho A R K_{z}\left(w_{j}-w_{w}\right)\left|\omega_{j}\right| \\
d_{\phi} & =\sum_{j=1}^{4}\left(\omega_{j} \rho A R^{2}\left(u_{j}-u_{w}\right) \frac{\sigma a}{2}\left(\frac{\theta_{0}}{3}-\frac{\lambda_{s t a t}}{4}\right)+\left|\omega_{j}\right| \rho A R\left(h K_{D}\left(v_{j}-v_{w}\right)\right.\right. \\
& \left.\left.-l K_{z}\left(w_{j}-w_{w}\right) \mathrm{s}_{j}\right)+\operatorname{sign}\left(\omega_{j}\right) \frac{1}{2} \rho A R K_{z}\left(u_{j}-u_{w}\right)\left(w_{j}-w_{w}\right)\right) \\
d_{\theta} & =\sum_{j=1}^{4}\left(\omega_{j} \rho A R^{2}\left(v_{j}-v_{w}\right) \frac{\sigma a}{2}\left(\frac{\theta_{0}}{3}-\frac{\lambda_{s t a t}}{4}\right)+\left|\omega_{j}\right| \rho A R\left(-h K_{D}\left(u_{j}-u_{w}\right)\right.\right. \\
& \left.\left.+l K_{z}\left(w_{j}-w_{w}\right) \mathrm{c}_{j}\right)+\operatorname{sign}\left(\omega_{j}\right) \frac{1}{2} \rho A R K_{z}\left(v_{j}-v_{w}\right)\left(w_{j}-w_{w}\right)\right) \\
& =\sum_{j=1}^{4}\left(\omega_{j} \rho A R^{2} K_{z}\left(w_{j}-w_{w}\right)\left(\frac{2 \theta_{0}}{3}-2 \lambda_{s t a t}\right)-\left|\omega_{j}\right| \rho l A R K_{D}\left(\left(v_{j}-v_{w}\right) \mathrm{c}_{j}\right.\right. \\
d_{\psi} & \left.\left(u_{j}-u_{w}\right) \mathrm{s}_{j}\right)-\operatorname{sign}\left(\omega_{j}\right) \rho A R\left(\frac{\sigma C_{D 0}}{8}\left(\left(u_{j}-u_{w}\right)^{2}+\left(v_{j}-v_{w}\right)^{2}\right)\right. \\
& \left.\left.\left.-w_{j}-w_{w}\right)^{2}\right)\right) \cdot
\end{aligned}
$$

\subsection{Disturbance upper bounds}

To design a control, which is able to compensate the disturbances, we have to evaluate the upper bounds for them.

For $x$ dynamics, the upper bound in body frame is computed from equation (10)

$$
\begin{aligned}
\left|d_{x}\right| & =\left|\tilde{K}_{D} \sum_{j=1}^{4}\left(u_{j}-u_{w}\right) \omega_{j}\right| \leq \tilde{K}_{D} \sum_{j=1}^{4}\left|u_{j}-u_{w}\right| \mid \omega_{j} \\
& \leq \tilde{K}_{D} \sum_{j=1}^{4}\left(\left|u_{j}\right|+\left|u_{w}\right|\right)\left|\omega_{j}\right| \leq \tilde{K}_{D}\left(\max _{j}\left|u_{j}\right|+\left|u_{w}\right|\right) \sum_{j=1}^{4}\left|\omega_{j}\right|
\end{aligned}
$$


where $\tilde{K}_{D}=\rho A R K_{D}$. Using the control $U_{z}=K_{f} \sum_{j=1}^{4} \omega_{j}^{2}$ and applying the Jensen's inequality, an upper estimate can be obtained

$$
\sum_{j=1}^{4}\left|\omega_{j}\right| \leq K \sqrt{\left|U_{z}\right|}, \quad K=\frac{2}{\sqrt{K_{f}}} .
$$

An upper bound of the disturbance becomes

$$
\left|d_{x}\right| \leq \bar{K}_{D}\left(|X|+D_{x}\right) \sqrt{\left|U_{z}\right|}=d_{x x}
$$

where $\bar{K}_{D}=K \tilde{K}_{D}$. In the earth frame using the rotation matrix (3) and the upper bound for the disturbances (17), (18), (19) it becomes

$$
\left|d_{x e}\right|=c_{\psi} c_{\theta} d_{x}+\left(c_{\psi} c_{\theta} c_{\phi}-s_{\psi} c_{\phi}\right) d_{y}+\left(s_{\phi} s_{\psi}+c_{\psi} s_{\theta} c_{\phi}\right) d_{z} \leq d_{x x}+2 d_{y y}+2 d_{z z}
$$

where $d_{y}, d_{z}, d_{y y}$ and $d_{z z}$ are derived below. For the disturbances $d_{y e}$ and $d_{z e}$ the computations are similar to the previous ones, therefore only final expressions are given next.

For $y$ dynamics, the upper bounds in body and earth frames respectively are computed from the equation (11)

$$
\begin{aligned}
\left|d_{y}\right| & \leq \bar{K}_{D}\left(|X|+D_{y}\right) \sqrt{\left|U_{z}\right|}=d_{y y} \\
\left|d_{y e}\right| & \leq d_{x x}+2 d_{y y}+2 d_{z z}
\end{aligned}
$$

For $z$ dynamics, the upper bounds in body and earth frames respectively are computed from the equation (12)

$$
\begin{aligned}
\left|d_{z}\right| & \leq \bar{K}_{z}\left(|X|+D_{z}\right) \sqrt{\left|U_{z}\right|}=d_{z z} \\
\left|d_{z e}\right| & \leq\left(f_{z e}(|X|)+D_{z e}\right) \sqrt{\left|U_{z}\right|} .
\end{aligned}
$$

where $\bar{K}_{z}=K \rho A R K_{z}, f_{z e}(|X|)=\bar{K}_{z} \max _{j}\left|w_{j}\right|+\bar{K}_{D} \max _{j}\left|u_{j}\right|+\bar{K}_{D} \max _{j}\left|v_{j}\right|$, $D_{z e}=\bar{K}_{z} D_{z}+\bar{K}_{D}\left(D_{x}+D_{y}\right)$.

For roll dynamics, the upper bound estimate is computed from the equations (13) and (16)

$$
\left|d_{\phi}\right| \leq \tilde{K}_{\phi}\left(f_{\phi 1}(X)+D_{\phi 1}\right) \sqrt{\left|U_{z}\right|}+\bar{K}_{\phi}\left(f_{\phi 2}(X)+D_{\phi 2}\right)
$$

where $K_{\phi 1}=\rho A R^{2} \frac{\sigma a}{2}\left(\frac{\theta_{0}}{3}-\frac{\lambda_{\text {stat }}}{4}\right), K_{\phi 2}=\rho A R h K_{D}, K_{\phi 3}=l K_{z} \rho A R, \tilde{K}_{\phi}=$ $K, f_{\phi 1}(X)=K_{\phi 1} \max _{j}\left|u_{j}\right|+K_{\phi 2} \max _{j}\left|v_{j}\right|+K_{\phi 3} \max _{j}\left|w_{j} \mathrm{~s}_{j}\right|, D_{\phi 1}=K_{\phi 1} D_{x}+$ 
$K_{\phi 2} D_{y}+K_{\phi 3} D_{z} \max _{j}\left|\mathrm{~s}_{j}\right|, \bar{K}_{\phi}=\frac{1}{2} \rho A R K_{z}, f_{\phi 2}(X)=\max _{j}\left|u_{j}\right|^{2}+\max _{j}\left|w_{j}\right|^{2}$, $D_{\phi 2}=D_{x}^{2}+D_{z}^{2}$.

For pitch dynamics, the upper bound estimate is computed from the equations (14) and (16)

$$
\left|d_{\theta}\right| \leq \tilde{K}_{\theta}\left(f_{\theta 1}(X)+D_{\theta 1}\right) \sqrt{\left|U_{z}\right|}+\bar{K}_{\theta}\left(f_{\theta 2}(X)+D_{\theta 2}\right)
$$

where $K_{\theta 1}=\rho A R^{2} \frac{\sigma a}{2}\left(\frac{\theta_{0}}{3}-\frac{\lambda_{s t a t}}{4}\right), K_{\theta 2}=\rho A R h K_{D}, K_{\theta 3}=l K_{z} \rho A R, \tilde{K}_{\theta}=$ $K, f_{\theta 1}(X)=K_{\theta 1} \max _{j}\left|v_{j}\right|+K_{\theta 2} \max _{j}\left|u_{j}\right|+K_{\theta 3} \max _{j}\left|w_{j} c_{j}\right|, D_{\theta 1}=K_{\theta 1} D_{y}+$ $K_{\theta 2} D_{x}+K_{\theta 3} D_{z} \max _{j}\left|\mathrm{c}_{j}\right|, \bar{K}_{\theta}=\frac{1}{2} \rho A R K_{z}, f_{\theta 2}(X)=\max _{j}\left|v_{j}\right|^{2}+\max _{j}\left|w_{j}\right|^{2}$, $D_{\theta 2}=D_{y}^{2}+D_{z}^{2}$.

For yaw dynamics, the upper bound estimate is computed from the equations (15) and (16)

$$
\left|d_{\psi}\right| \leq \tilde{K}_{\psi}\left(f_{\psi 1}(X)+D_{\psi 1}\right) \sqrt{\left|U_{z}\right|}+\bar{K}_{\psi}\left(f_{\psi 2}(X)+D_{\psi 2}\right)
$$

where $K_{\psi 1}=\rho A R^{2} K_{z}\left(\frac{2 \theta_{0}}{3}-2 \lambda_{s t a t}\right), K_{\psi 2}=\rho A R l K_{D}, K_{\psi 3}=\rho A R l K_{D}, \tilde{K}_{\psi}=$ $K, \quad f_{\psi 1}(X)=K_{\psi 1} \max _{j}\left|w_{j}\right|+K_{\psi 2} \max _{j}\left|v_{j} \mathrm{c}_{j}\right|+K_{\psi 3} \max _{j}\left|u_{j} \mathrm{~s}_{j}\right|, \quad D_{\psi 1}=$ $K_{\psi 1} D_{z}+K_{\psi 2} D_{y} \max _{j}\left|\mathrm{c}_{j}\right|+K_{\psi 3} D_{x} \max _{j}\left|\mathrm{~s}_{j}\right|, K_{\psi}=\rho A R, f_{\psi 2}(X)=\frac{\sigma C_{D 0}}{8}$ $\times\left(\max _{j}\left|u_{j}\right|^{2}+\max _{j}\left|v_{j}\right|^{2}\right)+\frac{4}{\sigma a} \max _{j}\left|w_{j}\right|^{2}, D_{\psi 2}=\frac{\sigma C_{D 0}}{8}\left(D_{x}^{2}+D_{y}^{2}\right)+\frac{4}{\sigma a} D_{z}^{2}$.

\section{Controls design}

For synthesis of control laws the SMC approach will be applied. First order and quasi-continuous SMCs (respectively 1-SMC, qc-SMC) will be designed in this section.

\subsection{First order control design}

This control methodology takes into account and compensates the matched disturbances. The big issue for the considered problem is that the disturbance $d$ depends on wind signals, the control itself, and state of the system, as shown above. Thus, a mild development of SMC approach is needed. To this end, the sliding surfaces in this work are selected proportional to the regulation errors $e_{i}$, in this way we are going to control the dynamics proportional to position and velocity

$$
S_{i}=\dot{e}_{i}+\alpha_{i} e_{i}, \quad \alpha_{i}>0,
$$


where $i \in(x, y, z, \phi, \theta, \psi)$. The Lyapunov function is then chosen as

$$
V_{i}=\frac{1}{2} S_{i}^{2}
$$

The dynamics of $z$ can be rewritten in the earth frame

$$
\ddot{z}=g-(\cos \phi \cos \theta) \frac{1}{m}\left(U_{z}+d_{z e}\right) .
$$

In hover state we have $\cos \theta \cos \phi \approx 1$, thus with a rotation of the UAV, a reasonable assumption is that $|\cos \phi \cos \theta| \geq \gamma>0$, where $\gamma$ is our operating point limit. To build the altitude control, the regulation error has been chosen as

$$
e_{z}=z-z_{\text {des }},
$$

where $z_{\text {des }}$ is the desired altitude for UAV. Thus, using (25) and its derivative in (22), the derivative of the sliding surface can be written as follows

$$
\dot{S}_{z}=\ddot{z}-\ddot{z}_{d e s}+\alpha_{z} \dot{e}_{z}
$$

and using (24), it also equals to

$$
\dot{S}_{z}=g-\frac{\cos \theta \cos \phi}{m}\left(U_{z}+d_{z e}\right)-\ddot{z}_{d e s}+\alpha_{z} \dot{e}_{z} .
$$

The following expression of the control can be selected

$$
U_{z}=\frac{m}{\cos \theta \cos \phi}\left(g-\tilde{u}_{z}-\ddot{z}_{\text {des }}+\alpha_{z} \dot{e}_{z}\right)
$$

where $\tilde{u}_{z}$ is an auxiliary control defined later. After substitution of the control (27) in (26) we obtain

$$
\dot{S}_{z}=\tilde{u}_{z}+d_{z e} \frac{\cos \theta \cos \phi}{m} .
$$

Using the Lyapunov function (23) with (28), its derivative takes the form

$$
\dot{V}=S_{z} \tilde{u}_{z}+S_{z} d_{z e} \frac{\cos \theta \cos \phi}{m} \leq S_{z} \tilde{u}_{z}+\left|S_{z}\right|\left|d_{z e} \frac{1}{m}\right| .
$$

Then, using (20), the Lyapunov derivative becomes

$$
\dot{V} \leq S_{z} \tilde{u}_{z}+\left|S_{z}\right| \frac{1}{m}\left(f_{z e}(|X|)+D_{z e}\right) \sqrt{\left|U_{z}\right|} .
$$


From the expression (27) we can derive

$$
\left|U_{z}\right| \leq \frac{m}{\gamma}\left(\left|g-\ddot{z}_{d e s}+\alpha_{z} \dot{e}_{z}\right|+\left|\tilde{u}_{z}\right|\right),
$$

and then, substituting in Lyapunov derivative, we obtain

$$
\dot{V} \leq S_{z} \tilde{u}_{z}+\left|S_{z}\right|\left(\varrho\left(X, \ddot{z}_{\text {des }}, \dot{z}_{\text {des }}\right)+\nu(X) \sqrt{\left|\tilde{u}_{z}\right|}\right) \text {, }
$$

where

$$
\begin{aligned}
\varrho\left(X, \ddot{z}_{\text {des }}, \dot{z}_{\text {des }}\right) & =\frac{1}{\sqrt{m \gamma}}\left(f_{z e}(|X|)+D_{z e}\right) \times \sqrt{\left|g+\alpha_{z} \dot{e}_{z}-\ddot{z}_{\text {des }}\right|}, \\
\nu(X) & =\frac{1}{\sqrt{m \gamma}}\left(f_{z e}(|X|)+D_{z e}\right) .
\end{aligned}
$$

For simplicity of notation, in the following part of the article we will denote $\varrho\left(X, \ddot{z}_{\text {des }}, \dot{z}_{\text {des }}\right)=\varrho(X)$. Let us look for the control in the form

$$
\tilde{u}_{z}=-\beta(X) \operatorname{sign}\left(S_{z}\right)
$$

where $\beta(X)$ is a function to synthesize. Substituting this control in the obtained inequality (30) we get

$$
\dot{V} \leq\left|S_{z}\right|(\varrho(X)+\nu(X) \sqrt{\beta(X)}-\beta(X))
$$

and it is necessary to ensure by a choice of $\beta(X)$

$$
\varrho(X)+\nu(X) \sqrt{\beta(X)}-\beta(X)=-\delta .
$$

Solving this quadratic inequality with respect to $\beta(X)$ we found

$$
\beta(X)=\frac{1}{2}\left(\nu(X)^{2}+2 \varrho(X)+\nu(X) \sqrt{\nu^{2}(X)+4 \varrho(X)}\right)+\delta,
$$

where $\delta>0$ is a tuning parameter. Substituting (31) in the inequality for $\dot{V}$ we get

$$
\dot{V}<-\sqrt{2} \delta \sqrt{V}
$$

consequently, the system state trajectory reaches and stays on the sliding surface $S_{z}$, which means asymptotic convergence of $e_{z}$ to the origin. Moreover, finite-time stability with respect to $S_{z}$ can be proved according to [2]. 
The dynamics of $x$ can be rewritten in the earth frame as follows

$$
\ddot{x}=(\sin \phi \sin \psi+\cos \psi \sin \theta \cos \phi) \frac{U_{z}}{m}-d_{x e} .
$$

Since $x$ dynamics cannot be directly controlled, a virtual control

$$
U_{x}=\sin \phi \sin \psi+\cos \psi \sin \theta \cos \phi
$$

is introduced, which will be used to find the desired Euler angles $\phi_{\text {des }}, \theta_{\text {des }}$ that will be the inputs of attitude controller next. Then the dynamics of $x$ takes the form

$$
\ddot{x}=U_{x} \frac{U_{z}}{m}-d_{x e} .
$$

In order to guarantee negativity of the time derivative of the Lyapunov function (23) the following expression of control in $x$ is selected

$$
U_{x}=\frac{m}{U_{z}}\left(\tilde{u}_{x}+\ddot{x}_{d e s}-\alpha_{x} \dot{e}_{x}\right),
$$

where

$$
\tilde{u}_{x}=-\left(d_{x x}+2 d_{y y}+2 d_{z z}\right) \operatorname{sign} S_{x}
$$

is an auxiliary SMC for $x$ dynamics. Such a design is admissible since the control $U_{z}$ is always separated with the zero and the discontinuous term in $\tilde{u}_{x}$, which leads to the chattering effect, is replaced with the continuous saturation approximation.

The $y$ dynamics, by introducing the virtual control

$$
U_{y}=\sin \psi \sin \theta \cos \phi-\cos \psi \sin \phi,
$$

can be rewritten in the earth frame as follows

$$
\ddot{y}=(\sin \psi \sin \theta \cos \phi-\cos \psi \sin \phi) \frac{U_{z}}{m}-d_{y e}=U_{y} \frac{U_{z}}{m}-d_{y e} .
$$

Using similar arguments as for $x$, the auxiliary SMC for $y$ dynamics takes the form

$$
\tilde{u}_{y}=-\left(d_{x x}+2 d_{y y}+2 d_{z z}\right) \operatorname{sign} S_{y},
$$

with the following expression of control in $y$

$$
U_{y}=\frac{m}{U_{z}}\left(\tilde{u}_{y}+\ddot{y}_{d e s}-\alpha_{y} \dot{e}_{y}\right) .
$$


The dynamics of $\phi$ can be rewritten as

$$
\dot{p}=q r \frac{I_{y y}-I_{z z}}{I_{x x}}+\frac{1}{I_{x x}}\left(U_{\phi}+d_{\phi}\right) .
$$

To build the roll control $U_{\phi}$, the error has been chosen as

$$
e_{\phi}=\phi-\phi_{d e s}
$$

Using (35) and substituting its derivative in (22), the derivative of the sliding surface can be calculated

$$
\dot{S}_{\phi}=\dot{p}-\ddot{\phi}_{d e s}+\alpha_{\phi} \dot{e}_{\phi}
$$

which due to (34) also equals to

$$
\dot{S}_{\phi}=q r \frac{I_{y y}-I_{z z}}{I_{x x}}+\frac{1}{I_{x x}}\left(U_{\phi}+d_{\phi}\right)+\alpha_{\phi} \dot{e}_{\phi}-\ddot{\phi}_{d e s} .
$$

The following expression of the control can be selected

$$
U_{\phi}=I_{x x}\left(-q r \frac{I_{y y}-I_{z z}}{I_{x x}}+\tilde{u}_{\phi}-\alpha_{\phi} \dot{e}_{\phi}+\ddot{\phi}_{d e s}\right),
$$

where $\tilde{u}_{\phi}$ is an auxiliary SMC. After substitution of this control in (36), we obtain

$$
\dot{S}_{\phi}=\tilde{u}_{\phi}+\frac{d_{\phi}}{I_{x x}}
$$

Using the Lyapunov function (23) with (37) and (21), its derivative becomes

$$
\begin{aligned}
\dot{V}= & S_{\phi} \tilde{u}_{\phi}+S_{\phi} d_{\phi} \leq S_{\phi} \tilde{u}_{\phi}+\left|S_{\phi}\right|\left|d_{\phi} \frac{1}{I_{x x}}\right| \leq S_{\phi} \tilde{u}_{\phi}+\frac{\left|S_{\phi}\right|}{I_{x x}} \\
& \times\left(\tilde{K}_{\phi}\left(f_{\phi 1}(X)+D_{\phi 1}\right) \sqrt{\left|U_{z}\right|}+\bar{K}_{\phi}\left(f_{\phi 2}(X)+D_{\phi 2}\right)\right) .
\end{aligned}
$$

To have (38) negative, the auxiliary control for $\phi$ dynamics must be

$$
\tilde{u}_{\phi}=-\frac{1}{I_{x x}} \operatorname{sign} S_{\phi}\left(\tilde{K}_{\phi}\left(f_{\phi 1}(X)+D_{\phi 1}\right) \sqrt{\left|U_{z}\right|}+\bar{K}_{\phi}\left(f_{\phi 2}(X)+D_{\phi 2}\right)\right) .
$$

Pitch and yaw controls can be designed following computations similar to the roll one, so only final expressions are given below. 
The dynamics of $\theta$ can be rewritten as

$$
\dot{q}=p r \frac{I_{z z}-I_{x x}}{I_{y y}}+\frac{1}{I_{y y}}\left(U_{\theta}+d_{\theta}\right) .
$$

The auxiliary control for $\theta$ dynamics

$$
\tilde{u}_{\theta}=-\frac{1}{I_{y y}} \operatorname{sign} S_{\theta}\left(\tilde{K}_{\theta}\left(f_{\theta 1}(X)+D_{\theta 1}\right) \sqrt{\left|U_{z}\right|}+\bar{K}_{\theta}\left(f_{\theta 2}(X)+D_{\theta 2}\right)\right),
$$

with the following expression of control

$$
U_{\theta}=I_{y y}\left(-p r \frac{I_{z z}-I_{x x}}{I_{y y}}+\tilde{u}_{\theta}-\alpha_{\theta} \dot{e}_{\theta}+\ddot{\theta}_{d e s}\right) .
$$

The dynamics of $\psi$ can be rewritten as

$$
\dot{r}=p q \frac{I_{x x}-I_{y y}}{I_{z z}}+\frac{1}{I_{z z}}\left(U_{\psi}+d_{\psi}\right) .
$$

The auxiliary control for $\psi$ dynamics

$$
\tilde{u}_{\psi}=-\frac{1}{I_{z z}} \operatorname{sign} S_{\psi}\left(\tilde{K}_{\psi}\left(f_{\psi 1}(X)+D_{\psi 1}\right) \sqrt{\left|U_{z}\right|}+\bar{K}_{\psi}\left(f_{\psi 2}(X)+D_{\psi 2}\right)\right),
$$

with the following expression of control

$$
U_{\psi}=I_{z z}\left(-p q \frac{I_{x x}-I_{y y}}{I_{z z}}+\tilde{u}_{\psi}-\alpha_{\psi} \dot{e}_{\psi}+\ddot{\psi}_{d e s}\right) .
$$

In the control design above it has been assumed that the rotors possess an immediate response on the desired values $\omega_{j}$ assigned to them by the control law. In reality they admit some dynamics, and for validation and comparison of the proposed control strategy, the transfer functions for the rotors have to be introduced in the realistic form as in eq. (40). Since it is a dynamics not considered during design, then it adds an undesired delay to the control. Recall also that the SMC is built for a simplified model with identified coefficients to diminish the design problem complexity, however it will be tested with the complete nonlinear UAV model validated through indoor experiments at ONERA lab.

Since a big shortage of SMC is the chattering (high frequency oscillations of a discontinuous control signal in the steady-state mode caused by imprecision 
of values of model parameters, digital and measurement noises), which can ruin the rotors, then below several solutions for chattering reduction are compared. To this end, several modifications are introduced into the control algorithm for a comparison.

In the literature the problem of chattering reduction is a well-known issue discussed in many articles, see for an example [7]. Saturation functions are popular solutions used for chattering reduction in SMC that leads to a practical stability in the closed-loop system. Arc-tangent function replaces the function sign with a smooth function:

$$
\operatorname{sat}_{\xi}(x)=\left\{\begin{array}{lll}
\operatorname{sign}(x) & \text { if } & |x| \geq \xi \\
\frac{4}{\pi} \arctan \left(\frac{x}{\xi}\right) & \text { if } & |x|<\xi
\end{array},\right.
$$

with the tunable gain $\xi>0$. According to [23], for a sufficiently small $\xi$ if for a sign function all trajectories converge in a finite time to an equilibrium, then with the saturation all trajectories will converge to a compact set around that equilibrium with the size proportional to the value of $\xi$. In practice $\xi$ should be chosen small enough to find a trade-off between chattering reduction and minimal acceptable steady-state error.

\subsection{Quasi-continuous control design}

Another way to reduce the chattering is to use a high order sliding mode (HOSM) control [2]. In this subsection for synthesis of a control law, a kind of HOSM control called the quasi-continuous SMC [24] will be applied, which can be considered as an approximation of the sign on the plane (see the Appendix for the explanation of the idea of qc-SMC). For this design the transfer functions for the rotors are taken into account in a generic and realistic form as previously in (9), which leads to additional dynamics

$$
b \dot{L}_{j}=U_{j}-L_{j} \quad j \in(z, \phi, \theta, \psi),
$$

where $L_{j}$ are the controls subjected by rotor dynamics.

Recall that for $z$ dynamics, the second derivative of the position error, $e_{z}=z-z_{\text {des }}$, has the form

$$
\ddot{e}_{z}=g-\frac{\cos \theta \cos \phi}{m}\left(L_{z}+d_{z e}\right)-\ddot{z}_{d e s},
$$


where the control $U_{z}$ is substituted by rotor dynamic output $L_{z}$ from (40). The second derivative of the position error can be rewritten

$$
\ddot{e}_{z}=-L_{z} \delta_{z}+\Delta_{z}
$$

where $\delta_{z}=(\cos \theta \cos \phi) / m, \Delta_{z}=g-\ddot{z}_{\text {des }}-\left(\cos \theta \cos \phi d_{z e}\right) / m$. The first derivative of the sliding surface is obtained

$$
\dot{S}_{z}=\ddot{e}_{z}+\alpha_{z} \dot{e}_{z}=-L_{z} \delta_{z}+\Delta_{z}+\alpha_{z} \dot{e}_{z}
$$

Using (40), its second derivative is computed as

$$
\ddot{S}_{z}=-\frac{U_{z}-L_{z}}{b} \delta_{z}+\dot{\delta}_{z} L_{z}+\dot{\Delta}_{z}+\alpha_{z}\left(-\delta_{z} L_{z}+\Delta_{z}\right)=-U_{z} \frac{\delta_{z}}{b}+\tilde{d}_{z},
$$

where $\tilde{d}_{z}=\dot{\Delta}_{z}+\alpha_{z}\left(-\delta_{z} L_{z}+\Delta_{z}\right)+\left(L_{z} \delta_{z}\right) / b+\dot{\delta}_{z} L_{z}$.

In Theorem 1 (see Appendix) it is stated that if the second order control is selected as

$$
U_{z}=\frac{b}{\delta_{z}} \tilde{D}_{z}(t, X) \frac{\left\lceil\dot{S}_{z}\right\rfloor^{2}+S_{z}}{\left|\dot{S}_{z}\right|^{2}+\left|S_{z}\right|}
$$

where $\lceil\cdot\rfloor^{2}=|\cdot|^{2} \operatorname{sign}(\cdot)$, then the point $S_{z}=\dot{S}_{z}=0$ is reached in a finite time provided that $\tilde{D}_{z}(t, X)>\left|\tilde{d}_{z}\right|$.

The position errors, $e_{x}=x-x_{d e s}, e_{y}=y-y_{d e s}$, have the dynamics

$$
\ddot{e}_{x}=U_{x} \frac{U_{z}}{m}-d_{x e}-\ddot{x}_{d e s}, \quad \ddot{e}_{y}=U_{y} \frac{U_{z}}{m}-d_{y e}-\ddot{y}_{d e s} .
$$

In [24] it is stated that if

$$
\ddot{e}_{x}=-\tilde{D}_{x}(t, X) \frac{\left\lceil\dot{e}_{x}\right\rfloor^{2}+\alpha_{x} e_{x}}{\left|\dot{e}_{x}\right|^{2}+\alpha_{x}\left|e_{y}\right|}, \quad \ddot{e}_{y}=-\tilde{D}_{y}(t, X) \frac{\left\lceil\dot{e}_{y}\right\rfloor^{2}+\alpha_{y} e_{y}}{\left|\dot{e}_{y}\right|^{2}+\alpha_{y}\left|e_{y}\right|},
$$

then $e_{i}=\dot{e}_{i}=0, i=x, y$ is reached in a finite time provided that $\tilde{D}_{x}(t, X)>$ $\left|d_{x e}+\ddot{x}_{d e s}\right|, \tilde{D}_{y}(t, X)>\left|d_{y e}+\ddot{y}_{\text {des }}\right| . x$ and $y$ dynamics are not influenced directly by the rotors, hence their stability does not need the introduction of an auxiliary sliding surface. The respective controls for $x, y$ positions are

$$
U_{x}=-\frac{m \tilde{D}_{x}(t, X)}{U_{z}} \frac{\left\lceil\dot{e}_{x}\right\rfloor^{2}+\alpha_{x} e_{x}}{\left|\dot{e}_{x}\right|^{2}+\alpha_{x}\left|e_{x}\right|}, \quad U_{y}=-\frac{m \tilde{D}_{y}(t, X)}{U_{z}} \frac{\left\lceil\dot{e}_{y}\right\rfloor^{2}+\alpha_{y} e_{y}}{\left|\dot{e}_{y}\right|^{2}+\alpha_{y}\left|e_{y}\right|}
$$


Such a design is admissible since the control $U_{z}$ is always separated with the zero and $U_{x}, U_{y}$ are always continuous by definition of the quasi-continuous control.

The controls for other dynamics can be designed following similar computations as for $z$, so only final expressions are given for roll, pitch and yaw, respectively. The second derivative of the position error, $e_{\phi}=\phi-\phi_{\text {des }}$, taking into account (40), has the form

$$
\ddot{e}_{\phi}=q r \frac{I_{y y}-I_{z z}}{I_{x x}}+\frac{1}{I_{x x}}\left(L_{\phi}+d_{\phi}\right)-\ddot{\phi}_{d e s},
$$

The roll control is

$$
U_{\phi}=-\frac{b}{\delta_{\phi}} \tilde{D}_{\phi}(t, X) \frac{\left\lceil\dot{S}_{\phi}\right\rfloor^{2}+S_{\phi}}{\left|\dot{S}_{\phi}\right|^{2}+\left|S_{\phi}\right|},
$$

where $\tilde{d}_{\phi}=\dot{\Delta}_{\phi}+\alpha_{\phi}\left(\delta_{\phi} L_{\phi}+\Delta_{\phi}\right)-\left(L_{\phi} \delta_{\phi}\right) / b-\dot{\delta}_{\phi} L_{\phi}, \delta_{\phi}=1 / I_{x x}, \Delta_{\phi}=$ $q r\left(I_{y y}-I_{z z}\right) / I_{x x}+d_{\phi} / I_{x x}-\ddot{\phi}_{d e s}$, with $\tilde{D}_{\phi}(t, X)>\left|\tilde{d}_{\phi}+\ddot{\phi}_{\text {des }}\right|$.

The second derivative of the position error, $e_{\theta}=\theta-\theta_{\text {des }}$, taking into account (40), has the form

$$
\ddot{e}_{\theta}=p r \frac{I_{z z}-I_{x x}}{I_{y y}}+\frac{1}{I_{y y}}\left(L_{\theta}+d_{\theta}\right)-\ddot{\theta}_{d e s},
$$

The pitch control is

$$
U_{\theta}=-\frac{b}{\delta_{\theta}} \tilde{D}_{\theta}(t, X) \frac{\left\lceil\dot{S}_{\theta}\right\rfloor^{2}+S_{\theta}}{\left|\dot{S}_{\theta}\right|^{2}+\left|S_{\theta}\right|},
$$

where $\tilde{d}_{\theta}=\dot{\Delta}_{\theta}+\alpha_{\theta}\left(\delta_{\theta} L_{\theta}+\Delta_{\theta}\right)-\left(L_{\theta} \delta_{\theta}\right) / b, \quad \delta_{\theta}=1 / I_{y y}, \quad \Delta_{\theta}=\operatorname{pr}\left(I_{z z}-\right.$ $\left.I_{x x}\right) / I_{y y}+d_{\phi} / I_{y y}-\ddot{\theta}_{d e s}$, with $\tilde{D}_{\theta}(t, X)>\left|\tilde{d}_{\theta}+\ddot{\theta}_{d e s}\right|$.

Recall that for $\psi$ dynamics, the second derivative of the position error, $e_{\psi}=\psi-\psi_{\text {des }}$, has the form

$$
\ddot{e}_{\psi}=p q \frac{I_{x x}-I_{y y}}{I_{z z}}+\frac{1}{I_{z z}}\left(L_{\psi}+d_{\psi}\right)-\ddot{\psi}_{d e s}
$$

The yaw control is

$$
U_{\psi}=-\frac{b}{\delta_{\psi}} \tilde{D}_{\psi}(t, X) \frac{\left\lceil\dot{S}_{\psi}\right\rfloor^{2}+S_{\psi}}{\left|\dot{S}_{\psi}\right|^{2}+\left|S_{\psi}\right|},
$$


where $\tilde{d}_{\psi}=\dot{\Delta}_{\psi}+\alpha_{\psi}\left(\delta_{\psi} L_{\psi}+\Delta_{\psi}\right)-\left(L_{\psi} \delta_{\psi}\right) / b, \delta_{\psi}=1 / I_{z z}, \Delta_{\psi}=p q\left(I_{x x}-\right.$ $\left.I_{y y}\right) / I_{z z}+d_{\psi} / I_{z z}-\ddot{\psi}_{\text {des }}$, with $\tilde{D}_{\psi}(t, X)>\left|\tilde{d}_{\psi}+\ddot{\psi}_{\text {des }}\right|$.

The proposed qc-SMC can be modified to counteract the chattering avoiding the saturation functions, and using the quasi-continuous function itself as an approximation of the sign on the plane, with a mild modification by adding a small constant $\varrho_{i}>0$ in the denominator:

$$
\frac{\left\lceil\dot{S}_{i}\right\rfloor^{2}+S_{i}}{\varrho_{i}+\left|\dot{S}_{i}\right|^{2}+\left|S_{i}\right|}, \quad \frac{\left\lceil\dot{e}_{i}\right\rfloor^{2}+\alpha_{i} e_{i}}{\varrho_{i}+\left|\dot{e}_{i}\right|^{2}+\alpha_{i}\left|e_{i}\right|} .
$$

where $\varrho_{i}$ is strictly related with accuracy. The smaller is $\varrho_{i}$ the higher is the effort on the rotors, which results in a more accentuated oscillation of the control, but with a smaller convergence error, and vice versa. According to [24], a finite-time convergence of the system can be achieved in the ideal case, when $\varrho_{i}=0$ and there is no measurement or implementation (digital) noises. In our case, since these restrictions are not satisfied, the convergence is assured with respect to a compact set around the desired trajectory. Then $\varrho_{i}$ are tuned accordingly to achieve a trade-off between control oscillations and convergence error.

Concluding the previous preliminary study, the quasi-continuous control is suitable to be applied in quadrotor regulation taking into account rotor dynamics without modification of the sliding surfaces, which have been designed for the conventional SMC without (9). It means that the function sign can be approximated efficacely with quasi-continuous function in SMC for quadrotors, and its finite time stability is proven in [24], considering $\varrho_{i}=0$. Then, considering the imposed quadrotor dynamics as in 1-SMC design $\left(\dot{e}_{i}+\alpha_{i} e_{i}=0\right.$ for $\left.i=x, y, z, \phi, \theta, \psi\right)$, the final expressions of qc-SMC chosen for implementation and comparison are given below.

The control for $z$ is given by

$$
\begin{aligned}
U_{z}= & \frac{m}{\cos \theta \cos \phi}\left(g-\ddot{z}_{\text {des }}+\alpha_{z} \dot{e}_{z}+\left(\frac { 1 } { 2 } \left(\nu(X)^{2}+2 \varrho(X)\right.\right.\right. \\
& \left.\left.\left.+\nu(X) \sqrt{\nu^{2}(X)+4 \varrho(X)}\right)+\frac{L_{z}}{b}-L_{z} \alpha_{z}+\varpi_{z}\right) \frac{\left\lceil\dot{S}_{z}\right\rfloor^{2}+S_{z}}{\varrho_{z}+\left|\dot{S}_{z}\right|^{2}+\left|S_{z}\right|}\right),
\end{aligned}
$$

where $\varpi_{z}>\left|\dot{\Delta}_{z}+\dot{\delta}_{z} L_{z}\right|$ is a tuning parameter.

The controls for $i=x, y$ are given by

$$
U_{i}=\frac{m}{U_{z}}\left(-\left(d_{x x}+2 d_{y y}+2 d_{z z}+\varpi_{i}\right) \frac{\left\lceil\dot{e}_{i}\right\rfloor^{2}+e_{i}}{\varrho_{i}+\left|\dot{e}_{i}\right|^{2}+\left|e_{i}\right|}+\ddot{i}_{d e s}-\alpha_{i} \dot{e}_{i}\right),
$$


where $\varpi_{i}>0$ is a tuning parameter with $\tilde{D}_{i}(t, X)>\left|d_{i e}+\ddot{i}_{\text {des }}\right|$.

The controls for roll, pitch and yaw respectively are given by

$$
\begin{aligned}
U_{\phi} & =I_{x x}\left(-q r \frac{I_{y y}-I_{z z}}{I_{x x}}+\tilde{u}_{\phi}-\alpha_{\phi} \dot{e}_{\phi}+\ddot{\phi}_{d e s}\right), \\
U_{\theta} & =I_{y y}\left(-p r \frac{I_{z z}-I_{x x}}{I_{y y}}+\tilde{u}_{\theta}-\alpha_{\theta} \dot{e}_{\theta}+\ddot{\theta}_{d e s}\right), \\
U_{\psi} & =I_{z z}\left(-p q \frac{I_{x x}-I_{y y}}{I_{z z}}+\tilde{u}_{\psi}-\alpha_{\psi} \dot{e}_{\psi}+\ddot{\psi}_{d e s}\right),
\end{aligned}
$$

where the auxiliary controls are defined as

$$
\begin{aligned}
\tilde{u}_{\phi}= & -\frac{1}{I_{x x}} \frac{\left\lceil\dot{S}_{\phi}\right\rfloor^{2}+S_{\phi}}{\varrho_{\phi}+\left|\dot{S}_{\phi}\right|^{2}+\left|S_{\phi}\right|}\left(\tilde{K}_{\phi}\left(f_{\phi 1}(X)+D_{\phi 1}\right) \sqrt{\left|U_{z}\right|}\right. \\
& \left.+\bar{K}_{\phi}\left(f_{\phi 2}(X)+D_{\phi 2}\right)+\varpi_{\phi}+\alpha_{\phi} \delta_{\phi} L_{\phi}-\frac{\delta_{\phi}}{b} L_{\phi}\right), \\
\tilde{u}_{\theta}= & -\frac{1}{I_{y y}} \frac{\left\lceil\dot{S}_{\theta}\right\rfloor^{2}+S_{\theta}}{\varrho_{\theta}+\left|\dot{S}_{\theta}\right|^{2}+\left|S_{\theta}\right|}\left(\tilde{K}_{\theta}\left(f_{\theta 1}(X)+D_{\theta 1}\right) \sqrt{\left|U_{z}\right|}\right. \\
& \left.+\bar{K}_{\theta}\left(f_{\theta 2}(X)+D_{\theta 2}\right)+\varpi_{\theta}+\alpha_{\theta} \delta_{\theta} L_{\theta}-\frac{\delta_{\theta}}{b} L_{\theta}\right), \\
\tilde{u}_{\psi}= & -\frac{1}{I_{z z}} \frac{\left\lceil\dot{S}_{\psi}\right\rfloor^{2}+S_{\psi}}{\varrho_{\psi}+\left|\dot{S}_{\psi}\right|^{2}+\left|S_{\psi}\right|}\left(\tilde{K}_{\psi}\left(f_{\psi 1}(X)+D_{\psi 1}\right) \sqrt{\left|U_{z}\right|}\right. \\
& \left.+\bar{K}_{\psi}\left(f_{\psi 2}(X)+D_{\psi 2}\right)+\varpi_{\psi}+\alpha_{\psi} \delta_{\psi} L_{\psi}-\frac{\delta_{\psi}}{b} L_{\psi}\right),
\end{aligned}
$$

with $\varpi_{i}>\left|\dot{\Delta}_{i}\right|, i=\phi, \theta, \psi$ tuning parameters.

Homogeneous differentiator [25] (see the expression (42) below) is used to estimate the value of the first derivative of the sliding surfaces $\dot{S}_{i}$.

\subsection{Physical constraints and tools}

Since UAVs are physical objects, then they are subjected to physical constraints, which correspond to their maximal linear and angular velocities and accelerations. Consequently, a smooth position trajectory is desired.

In literature many articles are presented to generate desired trajectories admissible to the system dynamics. Since the focus of the presented article is the control of a mini drone, then simple method is used which can take into account UAV physical constraints imposed during indoor experiments. The 
desired step references for coordinates $x, y, z$ are filtered (using a third order filter) to obtain realistic velocity and acceleration trajectories

$$
\frac{\xi_{f}}{\xi_{w p}}=\frac{1}{\left(1+\tau_{p o s} s\right)^{3}},
$$

where $\xi_{w p}$ is the way-point step reference, $\xi_{f}=\left(x_{d e s}, y_{d e s}, z_{d e s}\right)$ is the filtered signal, $\tau_{p o s}$ is the weight to create the desired smooth trajectory. The physical constraints are chosen

$$
\begin{gathered}
v_{\min }=\left[\begin{array}{c}
\dot{x}_{\min } \\
\dot{y}_{\min } \\
\dot{z}_{\min }
\end{array}\right], v_{\max }=\left[\begin{array}{c}
\dot{x}_{\max } \\
\dot{y}_{\max } \\
\dot{z}_{\max }
\end{array}\right] ; \\
a_{\min }=\left[\begin{array}{c}
\ddot{x}_{\min }=\frac{T_{\min }}{m} \sin \theta_{\min } \\
\ddot{y}_{\min }=\frac{T_{\min }}{m} \sin \phi_{\min } \\
\ddot{z}_{\min }=\frac{T_{\min }}{m}-g
\end{array}\right], a_{\max }=\left[\begin{array}{c}
\ddot{x}_{\max }=\frac{T_{\max }}{m} \sin \theta_{\max } \\
\ddot{y}_{\max }=\frac{T_{\max }}{m} \sin \phi_{\max } \\
\ddot{z}_{\max }=\frac{T_{\max }}{m}-g
\end{array}\right]
\end{gathered}
$$

where $T_{\max }$ is $90 \%$ of the maximal thrust achievable by the UAV to ensure that we still have some available thrust to proper control the drone in case of safe maneuvers; $T_{\min }$ is the minimal thrust. The reference angles $\phi_{\text {des }}, \theta_{\text {des }}$ are saturated in an admissible range of $\phi_{\min }, \theta_{\min }$ minimal values and $\phi_{\max }, \theta_{\max }$ maximal values, to avoid the singularities.

The desired angles $\phi_{\text {des }}, \theta_{\text {des }}$ are derived from the expressions for $U_{x}$ and $U_{y}$, using the desired value of $\psi$

$$
\begin{aligned}
& \phi_{\text {des }}=\arcsin \left(U_{x} \sin \psi_{\text {des }}-U_{y} \cos \psi_{\text {des }}\right), \\
& \theta_{\text {des }}=\arcsin \left(\frac{U_{x} \cos \psi_{\text {des }}+U_{y} \sin \psi_{\text {des }}}{\cos \phi_{\text {des }}}\right) .
\end{aligned}
$$

$U_{x}$ and $U_{y}$ are illustrated in equations (32), (33) with the saturation (39) for 1-SMC, and in the eq. (41) for qc-SMC. Physical constraints for angles and controls are chosen to be

$$
\Delta_{\min }=\left[\begin{array}{c}
\phi_{\min } \\
\theta_{\min } \\
\psi_{\min }
\end{array}\right], \quad \Delta_{\max }=\left[\begin{array}{c}
\phi_{\max } \\
\theta_{\max } \\
\psi_{\max }
\end{array}\right] ; \quad \Omega_{\min }=\left[\begin{array}{c}
\dot{\phi}_{\min } \\
\dot{\theta}_{\min } \\
\dot{\psi}_{\min }
\end{array}\right], \quad \Omega_{\max }=\left[\begin{array}{c}
\dot{\phi}_{\max } \\
\dot{\theta}_{\max } \\
\dot{\psi}_{\max }
\end{array}\right] ;
$$




$$
\begin{aligned}
& U_{\phi, \theta, \psi \min }=\left[\begin{array}{c}
-l \rho A R^{2} C_{\text {Tstat }} \sin (\epsilon)\left(\omega_{\max }^{2}-\omega_{\min }^{2}\right) \\
-l \rho A R^{2} C_{T s t a t} \cos (\epsilon)\left(\omega_{\text {max }}^{2}-\omega_{\text {min }}^{2}\right) \\
-2 \rho A R^{3} C_{Q}\left(\omega_{\text {max }}^{2}-\omega_{\min }^{2}\right)
\end{array}\right], \\
& U_{\phi, \theta, \psi \max }=\left[\begin{array}{c}
l \rho A R^{2} C_{\text {Tstat }} \sin (\epsilon)\left(\omega_{\text {max }}^{2}-\omega_{\text {min }}^{2}\right) \\
l \rho A R^{2} C_{\text {Tstat }} \cos (\epsilon)\left(\omega_{\text {max }}^{2}-\omega_{\text {min }}^{2}\right) \\
2 \rho A R^{3} C_{Q}\left(\omega_{\text {max }}^{2}-\omega_{\text {min }}^{2}\right)
\end{array}\right] .
\end{aligned}
$$

In order to implement the designed controls the first and the second derivatives of the desired angles have to be calculated, which are obtained using a homogeneous finite time differentiator, as in [25]

$$
\begin{gathered}
\dot{z}_{1}(t)=-\lambda_{1}\left\lceil z_{1}(t)-f(t)\right\rfloor^{\beta_{1}}+z_{2}(t), \quad \dot{z}_{2}(t)=-\lambda_{2}\left\lceil z_{1}(t)-f(t)\right\rfloor^{\beta_{2}}+z_{3}(t), \\
\dot{z}_{3}(t)=-\lambda_{3}\left\lceil z_{1}(t)-f(t)\right]^{\beta_{3}},
\end{gathered}
$$

where $z \in \mathbb{R}^{3}$ is the differentiator state and $f(t)$ is the signal to be differentiated, $\beta_{i}=1-j \tau$ for $j=1,2,3$ and any $\tau \in\left(-\frac{1}{3}, 0\right)$, while the parameters $\lambda_{1}, \lambda_{2}, \lambda_{3}$ are selected to ensure the Hurwitz property of the matrix

$$
A=\left[\begin{array}{lll}
-\lambda_{1} & 1 & 0 \\
-\lambda_{2} & 0 & 1 \\
-\lambda_{3} & 0 & 0
\end{array}\right] .
$$

Then the system (42) performs estimation of the first and second derivative of $f(t)$ in a finite time: $\hat{\dot{f}}(t)=z_{2}(t), \hat{\vec{f}}(t)=z_{3}(t)$. Increasing the smallest eigenvalue of $A$ improves the rate of convergence. In this work $\tau<0$ is sufficiently big, the eigenvalues of $A$ are chosen by trial and error accordingly to the desired dynamics and in such a way to avoid undesired response delay.

\subsection{Control design summary}

The generic scheme of hierarchical control algorithm presented above is given in Fig. 3. Homogeneous differentiator is used to estimate the first and second derivatives of angles and the first derivative of the sliding surfaces. The stability of the system can always be proved respecting the maximal value of $\phi, \theta, D_{x}, D_{y}, D_{z}$. In the proposed algorithms, the control gains are functions of the state and wind velocity, then using a wind estimator the wind velocity estimates can be substituted on-line in the control law, as in Fig. 4. In this way, by adapting the control amplitude, the regulator effort on the rotors can be reduced when it is possible. A good wind estimator is desired to reduce the uncertainty that will affect directly the performance of the proposed control. However, in this paper maximal admissible wind values are considered as input to the control. 


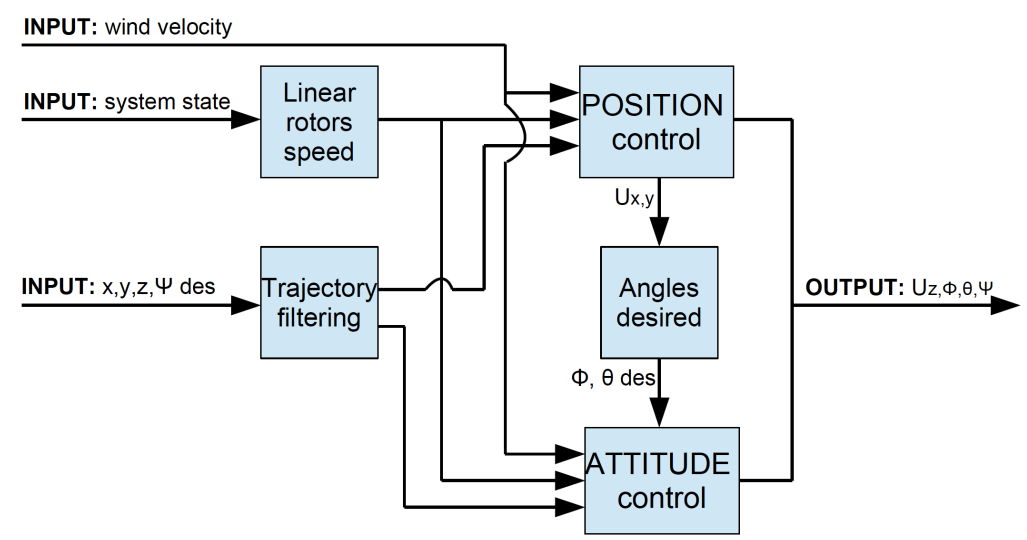

Figure 3: Hierachical control scheme

\section{Numerical Validation}

Numerical experiments are performed in the simulator using UAV Simulink library, built with nonlinear aerodynamic coefficient, and using the rotor model in eq. (4). The quadrotor model was developed using blade momentum theory in aerodynamic science and next validated through indoor experiments with the commercial Parrot Ar Drone 2.0 at low/moderate velocity at the ONERA Aerodynamics Aeroelasticity Acoustics Department. Wind perturbations, affecting the UAV nonlinear model, are simulated as sinusoids, which is a reasonable assumption since the gust generator at ONERA lab allows to create a sinusoidal wind gust profile, hence the sinusoid wind effect over time is correlated to the motion of the drone through the turbulent area. For simplicity of demonstration the wind velocities are selected as constant input in qc-SMC and in 1-SMC the wind velocities are replaced with their constant upper bounds, see Fig. 5 (wind $\mathrm{x}$, wind $\mathrm{y}$, wind $\mathrm{z}$ ). This restriction is imposed in the article to better present the new design strategy. Conventional SMC (conv-SMC), used for comparisons, is built as $\tilde{u}_{i}=C_{i} \operatorname{sign} S_{i}$ for constant $C_{i} \geq\left\|d_{i}\right\|_{\infty}, i=x, y, z, \phi, \theta, \psi$ and suitable under a popular hypothesis that perturbations in the basic quadrotor model are bounded functions of time. In conv-SMC, as usual for implementation, the function sign is replaced by the approximation $\mathrm{sat}_{\xi_{i}}$ in eq. (39). Constant gains $C_{i}$ are tuned to compensate the disturbances (dist $\mathrm{x}$, dist $\mathrm{y}$, dist $\mathrm{z}$, dist $\phi$, dist $\theta$, dist $\psi$ ) in Fig. 5 and taking their maximal values. Since three nonlinear controllers are studied, a rule is imposed to make the comparisons: the same performance of the 


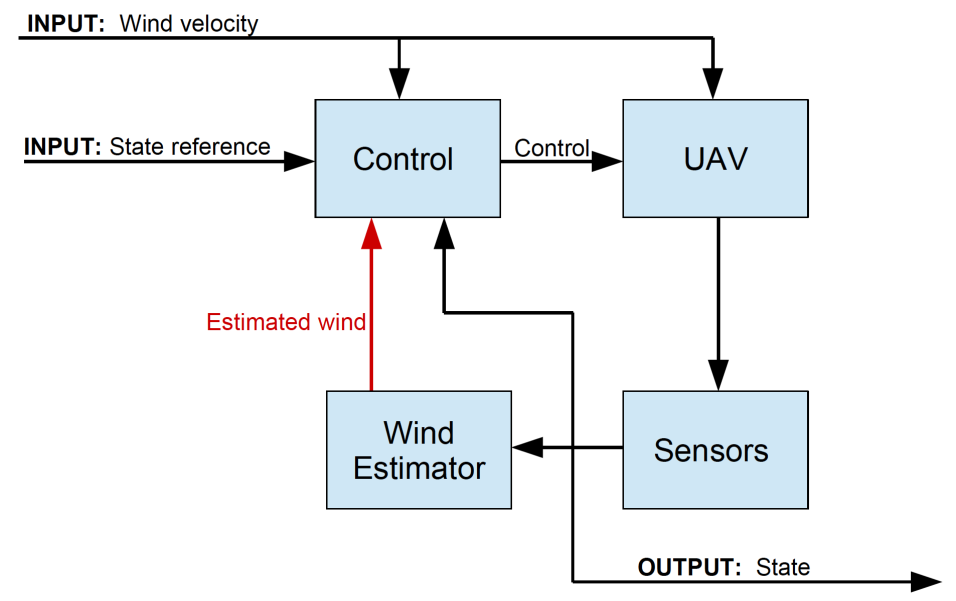

Figure 4: Control-wind estimator coupling. The red arrow indicates how varying wind estimation can be merged with the control.

controllers are imposed by tuning and keeping the same $\xi_{i}$ for conv-SMC and 1-SMC, $\varrho_{i}$ are adjusted to have a similar performance for the qc-SMC, and same values of $\alpha_{i}$ are also considered. Unfortunately, 1-SMC and conv-SMC cannot achieve the same accuracy as the qc-SMC since they become unstable due to the high control oscillations if the convergence error is very small. For this reason, a second try is done keeping the same $\xi_{i}$ for 1-SMC and convSMC and "relaxing" them (changing $\xi_{i}$ to have bigger position convergence error), while a more accuracy is achieved for qc-SMC. Hence, we consider the values listed in Tables $1,3,2,4$ where $\dot{z}_{\max }, z_{\max }$ are with negative signs because $z$ axes is taken with the positive direction towards down as in Fig. 1. For simulations, a generic flight case is illustrated which couples together forward, lateral and vertical flights, moving the UAV in $x, y, z$ positions. The corresponding external disturbances, influenced by the proposed varying gain functions, and wind are illustrated in Fig. 5, where wind in $z$ is chosen small because of the small UAV mass and limited thrust. Figures 6, 7, 8, 9, 10, 11 illustrate the contribution of the controls together with state variables and reference states. After the presentation of the working hypothesis, the following conclusions are identified after these numeric experiments:

First, the simulation clearly shows that using a varying-gain function instead of a constant gain improves the transients, because the SMCs using this function are subjected to less effort on the rotors. Under the same $\xi_{i}$, conv-SMC is slightly more robust than the 1-SMC due to the fact that in 
the tuned constant gain of conv-SMC some inner dynamics are automatically considered (derivative of the control $U_{z}$, derivative of the wind velocity, etc...) but at the price of much higher control oscillation. This fact is due to the simplifications adopted in the disturbances design in sec. 3.1. However, more accuracy can be achieved also for the 1-SMC, changing the parameter $\xi_{i}$. While the tuned gain for the conv-SMC has to be done "a posteriori", the varying-gain of 1-SMC and of qc-SMC can be tuned "a priori" making an hypothesis on maximal wind velocities. This first point shows the effectiveness of the first contribution: tuning the varying-gains influenced by the state, the control itself, and the wind velocity.

Second, as already discussed, 1-SMC and conv-SMC tend to become unstable if higher accuracy (changing $\xi_{i}$ ) is imposed because the rotors dynamics is not considered in their designs. qc-SMC can achieve better accuracy among all the controls ensuring an acceptable control oscillation which is similar to the control in 1-SMC, but the resulted gain tuning is far to be optimal. Then, the trajectory can be reached changing freely $\varrho_{i}$ and enforcing the control to be more robust as in Figures 12, 13 without compromising the stability. This second point shows the effectiveness of the second contribution: the importance of including rotors dynamics inside the control design.

Last, regarding the qc-SMC, the error convergence of the system is affected by the introduction of $\varrho_{i}$ in the denominator of quasi-continuous function. Reducing the convergence error, increases the control oscillations, hence a good trade-off between effectiveness of the control and the effort on the rotors has to be found. However, thanks to the varying-gains built in subsection 3.2, a wind estimator can be coupled with the control, then wind velocity can be substituted on-line. In this way, by adapting the control amplitude, the regulator effort on the rotors can be reduced when it is possible, allowing to achieve a better performance with less control oscillations. This last point highlights the importance of the first contribution: tuning the varying-gains influenced by the wind velocity.

As a result, the presented simulations demonstrate the stability of the system affected by wind velocity and realistic external disturbances, respecting the limitations of the mini drone mass and thrust. 
Table 1: Parrot drone parameters

\begin{tabular}{|c|c|c|c|c|c|c|c|}
\hline Par. & $R$ & $l$ & $h$ & $g$ & $\theta_{0}$ & $m$ & $I_{x x}$ \\
Val. & 0.1 & 0.185 & -0.0125 & 9.81 & 23.9 & 0.472 & 0.00356 \\
Unit & $m$ & $m$ & $m$ & $m / s^{2}$ & $d e g$ & $K g$ & $K g . m^{2}$ \\
\hline \hline Par. & $I_{y y}$ & $I_{z z}$ & $\rho$ & $\sigma$ & $a$ & $C_{D 0}$ & $\lambda_{\text {stat }}$ \\
Val. & 0.00402 & 0.00712 & 1.25 & 0.111 & 4.6542 & 2.15 & 0.1056 \\
Unit & $K g . m^{2}$ & $K g . m^{2}$ & $K g / m^{3}$ & & & & \\
\hline \hline Par. & $C_{T s t a t}$ & $K_{D}$ & $K_{z}$ & & & & \\
Val. & 0.0223 & 0.06 & 0.09 & & & & \\
Unit & & & & &
\end{tabular}

Table 2: Controls saturation

\begin{tabular}{|c|c|c|c|c|}
\hline & $U_{z}(N)$ & $U_{\phi}(N m)$ & $U_{\theta}(N m)$ & $U_{\psi}(N m)$ \\
$\min$. & 1.4 & -0.14 & -0.14 & -0.30 \\
$\max$. & 5.6 & 0.14 & 0.14 & 0.30 \\
\hline
\end{tabular}

Table 3: Saturation parameters

\begin{tabular}{|c|c|c|c|c|c|c|c|c|}
\hline $\begin{array}{l}\text { Par. } \\
\text { Val. } \\
\text { Unit }\end{array}$ & $\begin{array}{c}\phi_{\max } \\
45 \\
\text { deg } \\
\end{array}$ & $\begin{array}{c}\theta_{\max } \\
45 \\
\operatorname{deg} \\
\end{array}$ & $\begin{array}{c}\psi_{\max } \\
180 \\
\text { deg }\end{array}$ & $\begin{array}{l}\phi_{\min } \\
-45 \\
\operatorname{deg} \\
\end{array}$ & $\begin{array}{l}\theta_{\min } \\
-45 \\
\operatorname{deg} \\
\end{array}$ & $\begin{array}{c}\psi_{\min } \\
-180 \\
\operatorname{deg}\end{array}$ & $\begin{array}{c}\dot{\phi}_{\max } \\
40 \\
\operatorname{deg} / s \\
\end{array}$ & $\begin{array}{c}\dot{\theta}_{\max } \\
40 \\
\operatorname{deg} / s \\
\end{array}$ \\
\hline Par. & $\dot{\psi}_{\max }$ & $\dot{\phi}_{\min }$ & $\dot{\theta}_{\min }$ & $\dot{\psi}_{\min }$ & $x_{\max }$ & $y_{\max }$ & $z_{\max }$ & $x_{\min }$ \\
\hline Val. & 10 & -40 & -40 & -10 & 100 & 100 & -100 & 0 \\
\hline Unit & $\operatorname{deg} / s$ & $\operatorname{deg} / s$ & $\operatorname{deg} / s$ & $\operatorname{deg} / s$ & $m$ & $m$ & $m$ & $m$ \\
\hline Par. & $y_{\text {min }}$ & $z_{\text {min }}$ & $\dot{x}_{\max }$ & $\dot{y}_{\max }$ & $\dot{z}_{\max }$ & $\dot{x}_{\min }$ & $\dot{y}_{\text {min }}$ & $\dot{z}_{\text {min }}$ \\
\hline Val. & 0 & 0 & 5 & 5 & -1 & -5 & -5 & 1 \\
\hline Unit & $m$ & $m$ & $\mathrm{~m} / \mathrm{s}$ & $\mathrm{m} / \mathrm{s}$ & $\mathrm{m} / \mathrm{s}$ & $\mathrm{m} / \mathrm{s}$ & $\mathrm{m} / \mathrm{s}$ & $\mathrm{m} / \mathrm{s}$ \\
\hline Par. & $\omega_{\max }$ & $\omega_{\min }$ & & & & & & \\
\hline Val. & 400 & 200 & & & & & & \\
\hline Unit & $\mathrm{rad} / \mathrm{s}$ & $\mathrm{rad} / \mathrm{s}$ & & & & & & \\
\hline
\end{tabular}

\section{Conclusion}

In this work, a robust sliding mode control approach is used to stabilize a small quadrotor UAV under wind perturbations. It is considered that the disturbance bound for a UAV quadrotor at a low/moderate velocity depends on the control itself, the wind speed and the state of the UAV. To this end, 
Table 4: Controls parameters

\begin{tabular}{|c|c|c|c|c|c|c|c|c|c|c|c|c|c|}
\hline Par. & $\alpha_{x}$ & $\alpha_{y}$ & $\alpha_{z}$ & $\alpha_{\phi}$ & $\alpha_{\theta}$ & $\alpha_{\psi}$ & $\xi_{x}$ & $\xi_{y}$ & $\xi_{z}$ & $\xi_{\phi}$ & $\xi_{\theta}$ & $\xi_{\psi}$ & $\varrho_{x}$ \\
Val. & 1 & 1 & 1 & 10 & 10 & 10 & 1 & 1 & 1 & 1.3 & 1.3 & 1.3 & 0.2 \\
\hline \hline Par. & $\varrho_{y}$ & $\varrho_{z}$ & $\varrho_{\phi}$ & $\varrho_{\theta}$ & $\varrho_{\psi}$ & $\varpi_{x}$ & $\varpi_{y}$ & $\varpi_{z}$ & $\varpi_{\phi}$ & $\varpi_{\theta}$ & $\varpi_{\psi}$ & $D_{x}$ & $D_{y}$ \\
Val. & 0.2 & 1 & 0.7 & 0.7 & 0.7 & 1 & 1 & 1 & 7 & 7 & 7 & 3 & 3 \\
\hline \hline Par. & $D_{z}$ & $C_{x}$ & $C_{y}$ & $C_{z}$ & $C_{\phi}$ & $C_{\theta}$ & $C_{\psi}$ & \multicolumn{1}{|c|}{} & & & & \\
Val. & 0.2 & 4 & 4 & 15 & 20 & 20 & 40 & & & & & & \\
\hline
\end{tabular}
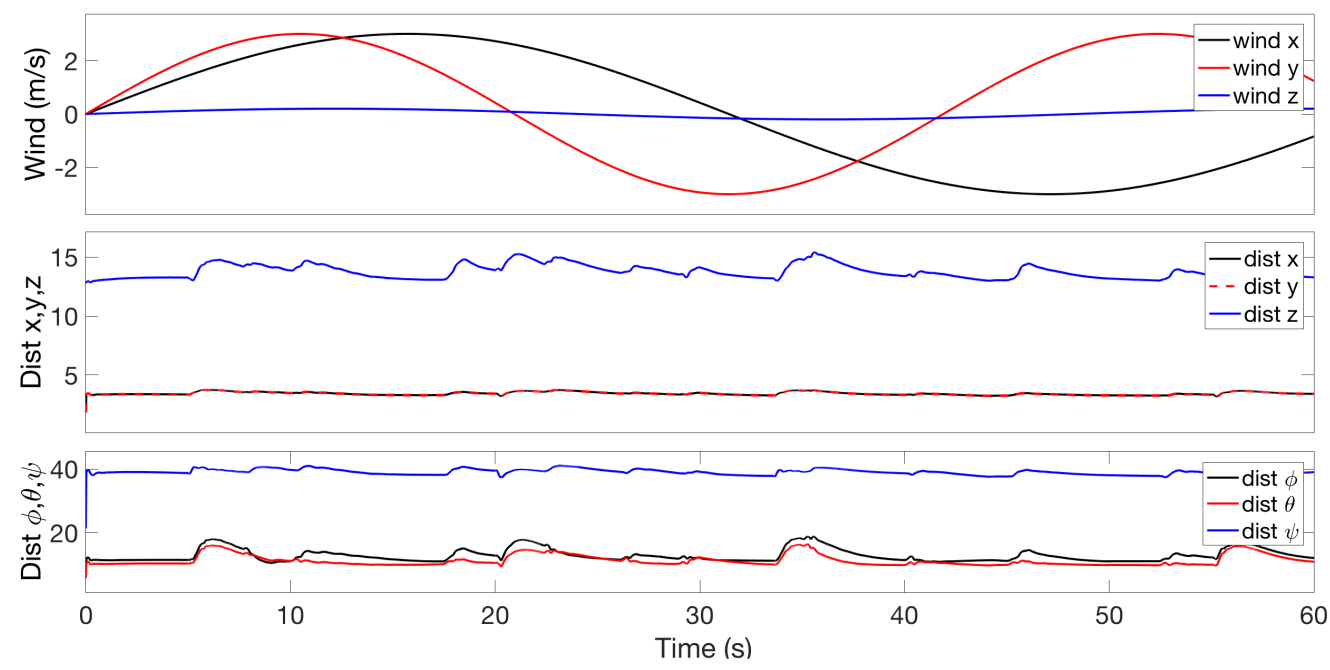

Figure 5: Wind velocities and the respective disturbances acting on $x, y, z, \phi, \theta, \psi$.

the constant gain, which is proportional to the sign functions in conventional sliding mode controls, is replaced with a time-varying function. In this way we aim to reduce as much as possible the control effort on the rotors, adapting in real-time the weighted function used in the control design.

The issue originated by introduction of the rotor transfer functions is studied and several solutions for decreasing chattering are applied. Then, the contribution with respect to conventional first order sliding mode control is highlighted, and a recent quasi-continuous high order sliding mode control is also tested and discussed in simulations.

The presented results of numerical experiments confirm the effectiveness of the designed control to stabilize the drone under varying wind, and its stability is proved for a known maximal admissible wind value. 

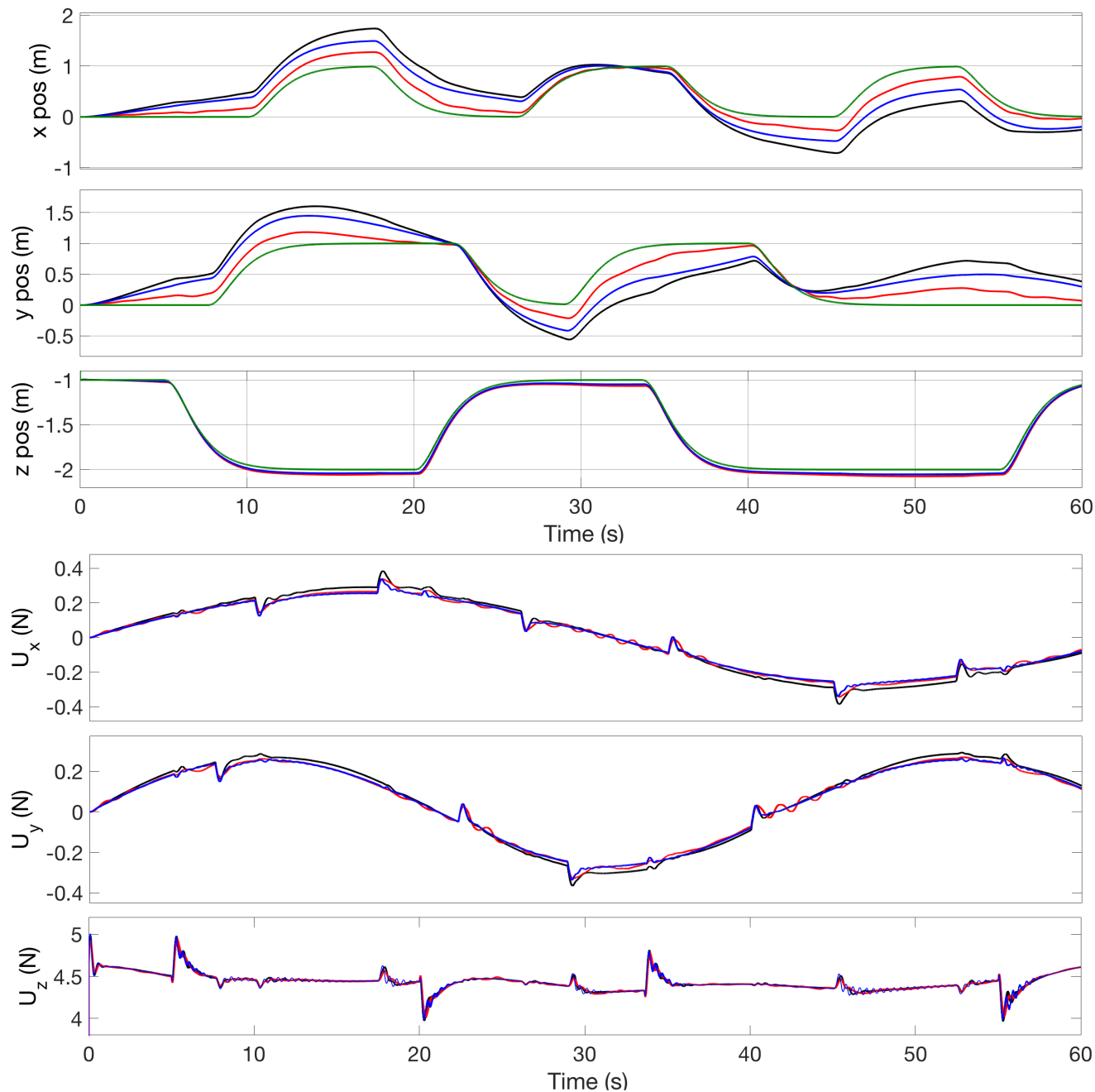

Figure 6: $x, y, z$ position and respective controls ( - : desired trajectory; $-:$ 1-SMC; - : conv-SMC; - : qc-SMC). 

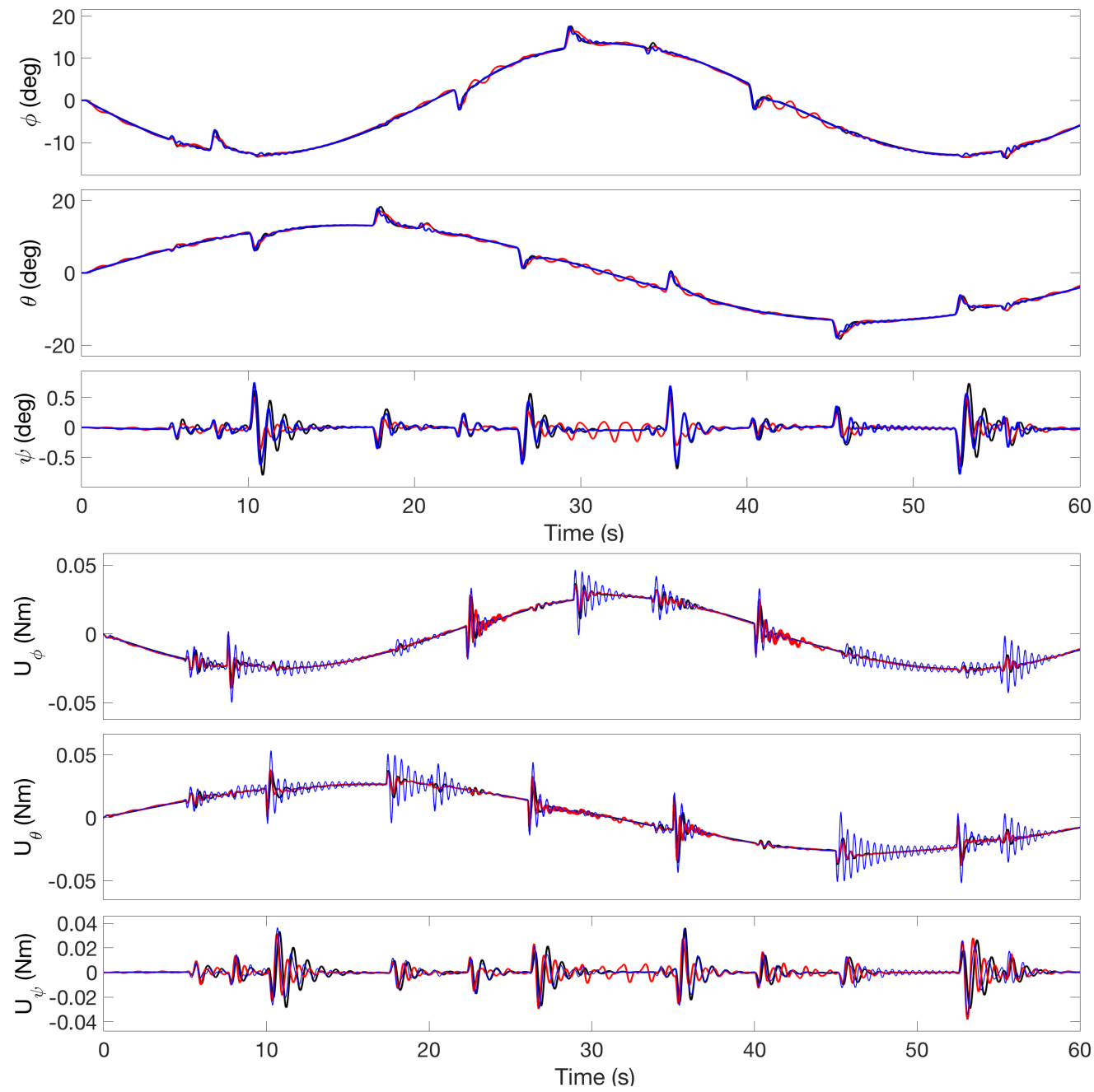

Figure 7: $\phi, \theta, \psi$ angles and respective controls (—: 1-SMC; - : conv-SMC; —: qc-SMC). 

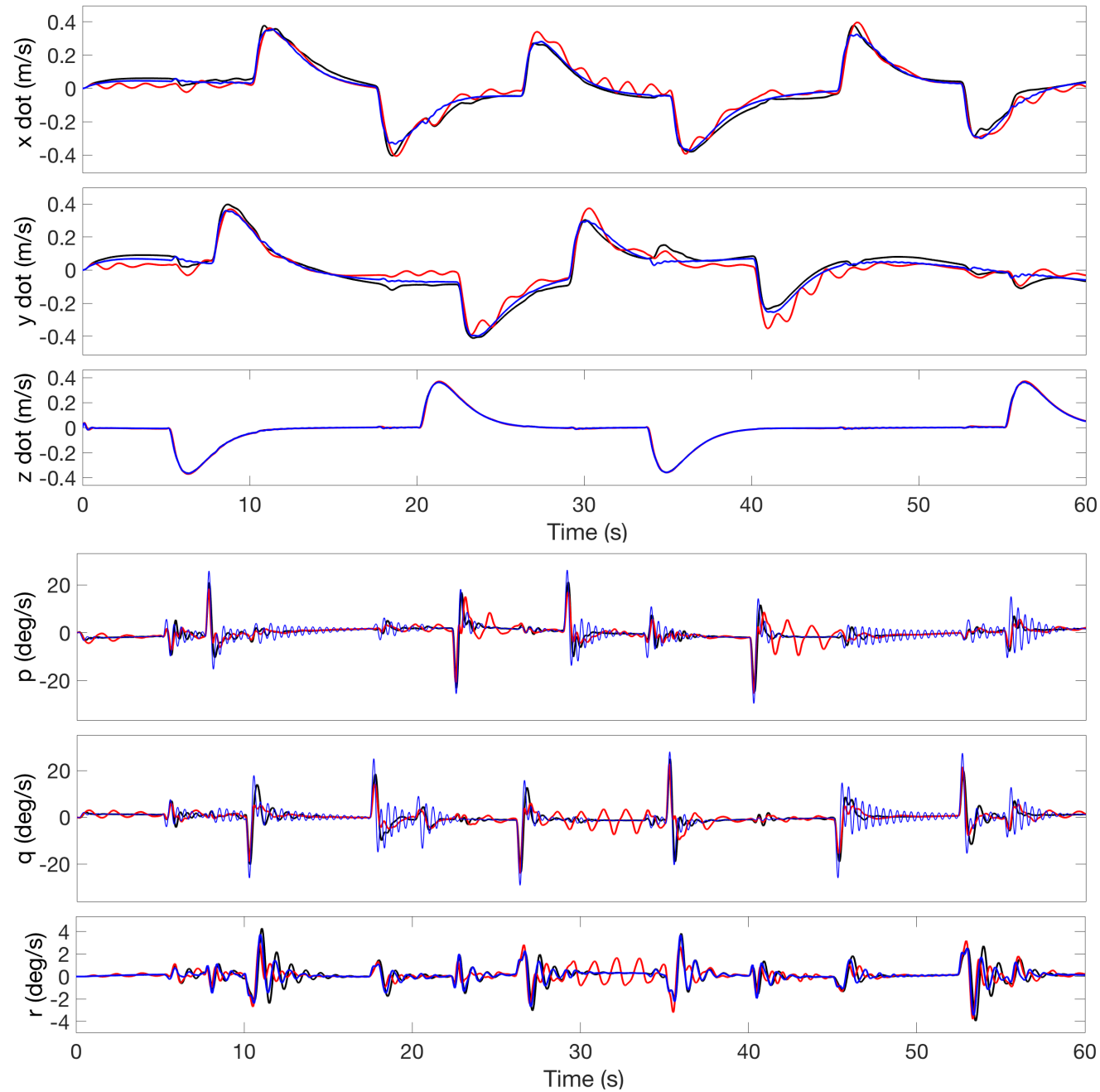

Figure 8: Linear and angular velocities (—: 1-SMC; —: conv-SMC; —: qc-SMC). 


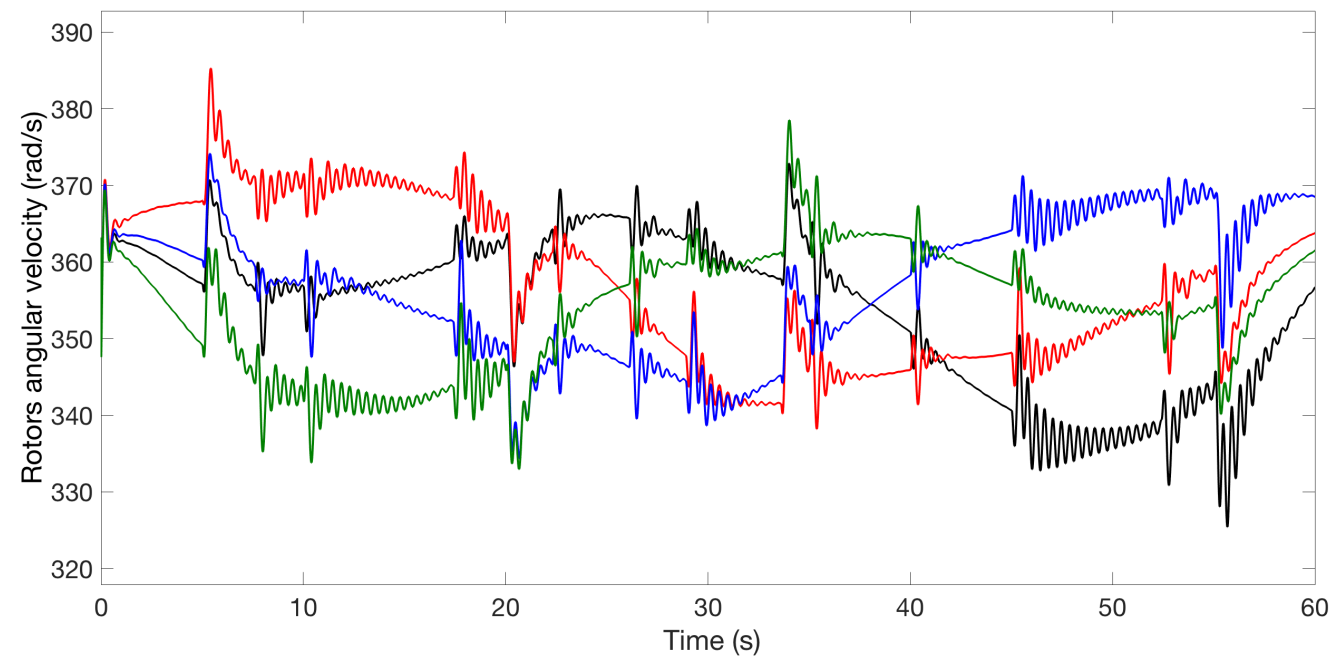

Figure 9: Rotors angular velocities for conventional SMC.

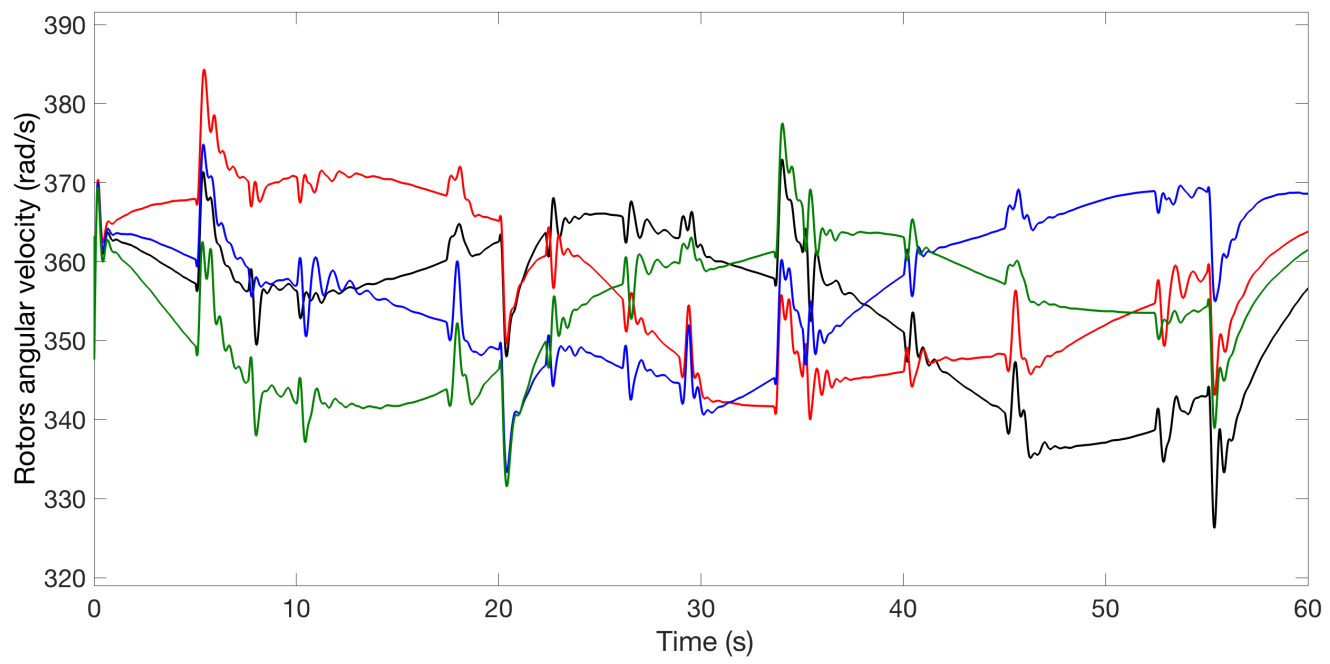

Figure 10: Rotors angular velocities for 1-SMC. 


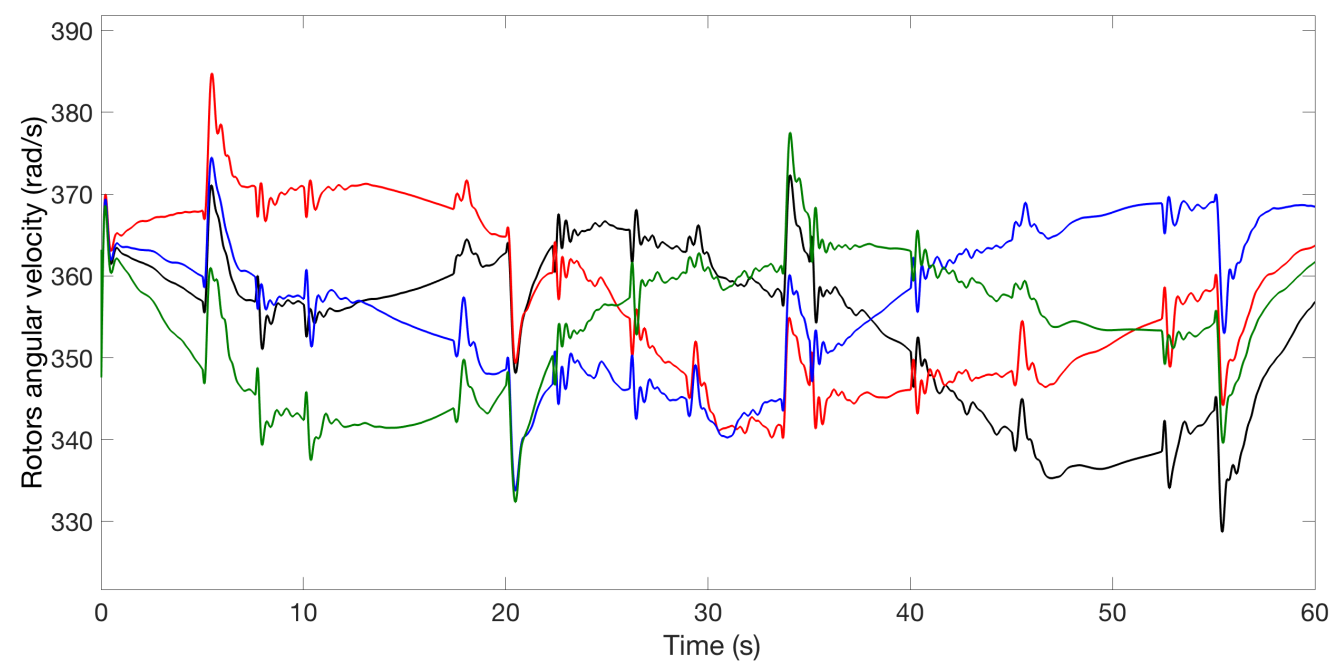

Figure 11: Rotors angular velocities for qc-SMC.

In this work constant maximal admissible wind values are considered as direct input to the control. However, since this approach is strictly related to the wind estimation, the coupling with the estimator, as in Fig. 4 is desired. Further works lead to a deeper study of their interaction, which must ensure the stability of the system in a compact set around the equilibrium depending by the estimation incertitude.

\section{Appendix A. 1}

Following [24] consider a double integrator system:

$$
\ddot{x}(t)=g(t, X(t)) u(t)+h(t, X(t)),
$$

where $X(t)=[x(t), \dot{x}(t)]^{T} \in \mathbb{R}^{2}$ is the state vector, $u(t) \in \mathbb{R}$ is the control input, two functions $g: \mathbb{R}^{3} \rightarrow \mathbb{R}$ and $h: \mathbb{R}^{3} \rightarrow \mathbb{R}$ ensure forward existence and uniqueness of the system solutions at least locally. In addition, there are two known functions $\underline{g}: \mathbb{R}^{3} \rightarrow \mathbb{R}$ and $\bar{h}: \mathbb{R}^{3} \rightarrow \mathbb{R}$ such that for all $X \in \mathbb{R}^{2}$ and $t \geq 0$

$$
g(t, X) \geq \underline{g}(t, X)>0,|h(t, X)| \leq \bar{h}(t, X) .
$$

The following control for quasi-continuous SMC can be proposed for (A.1) (a more generic case is studied in [24] where bounded time-varying positive 

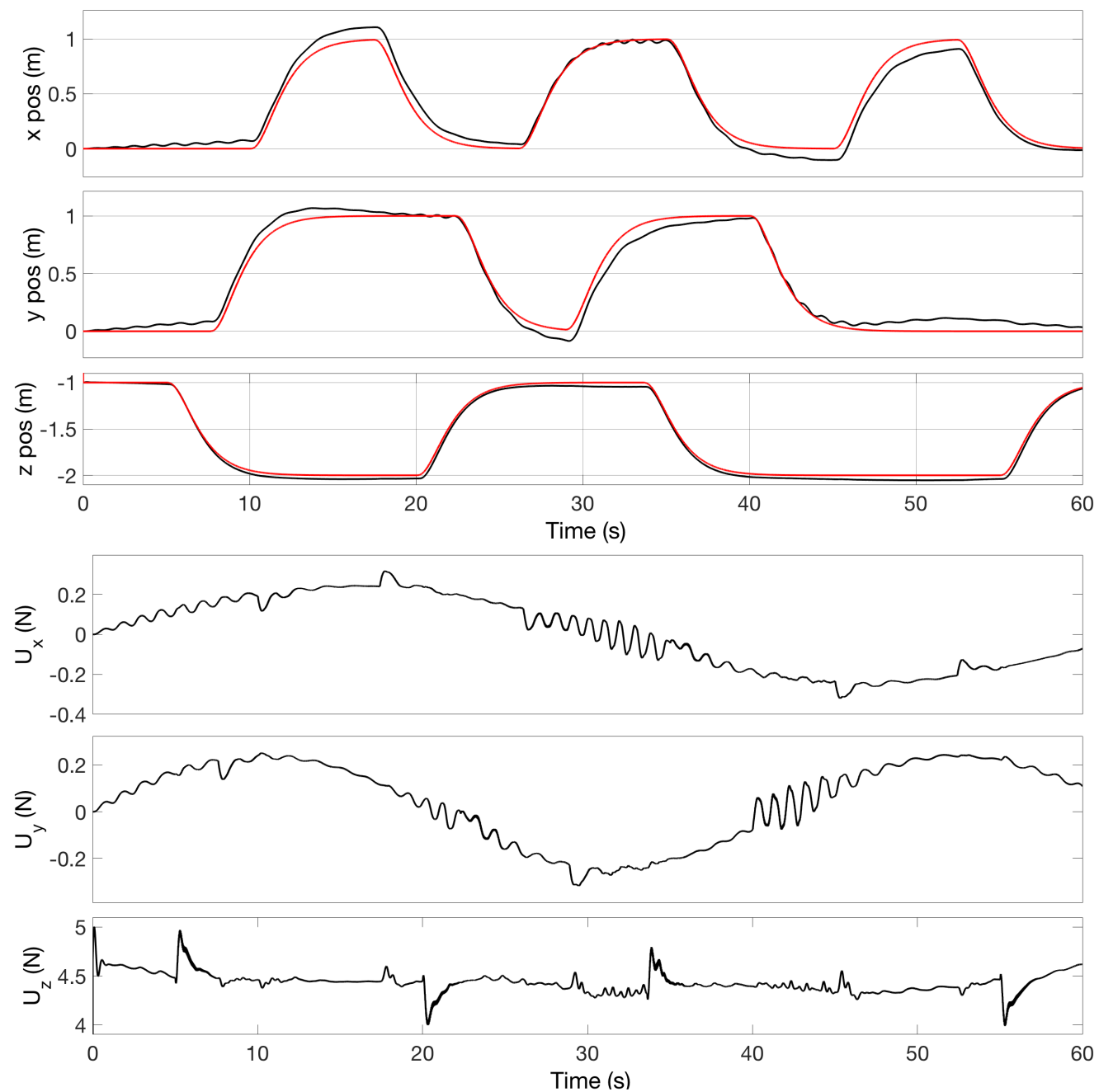

Figure 12: $x, y, z$ position and respective controls $\left(-\right.$ : qc-SMC changing $\varrho_{i} ;-$ : desired trajectory). 

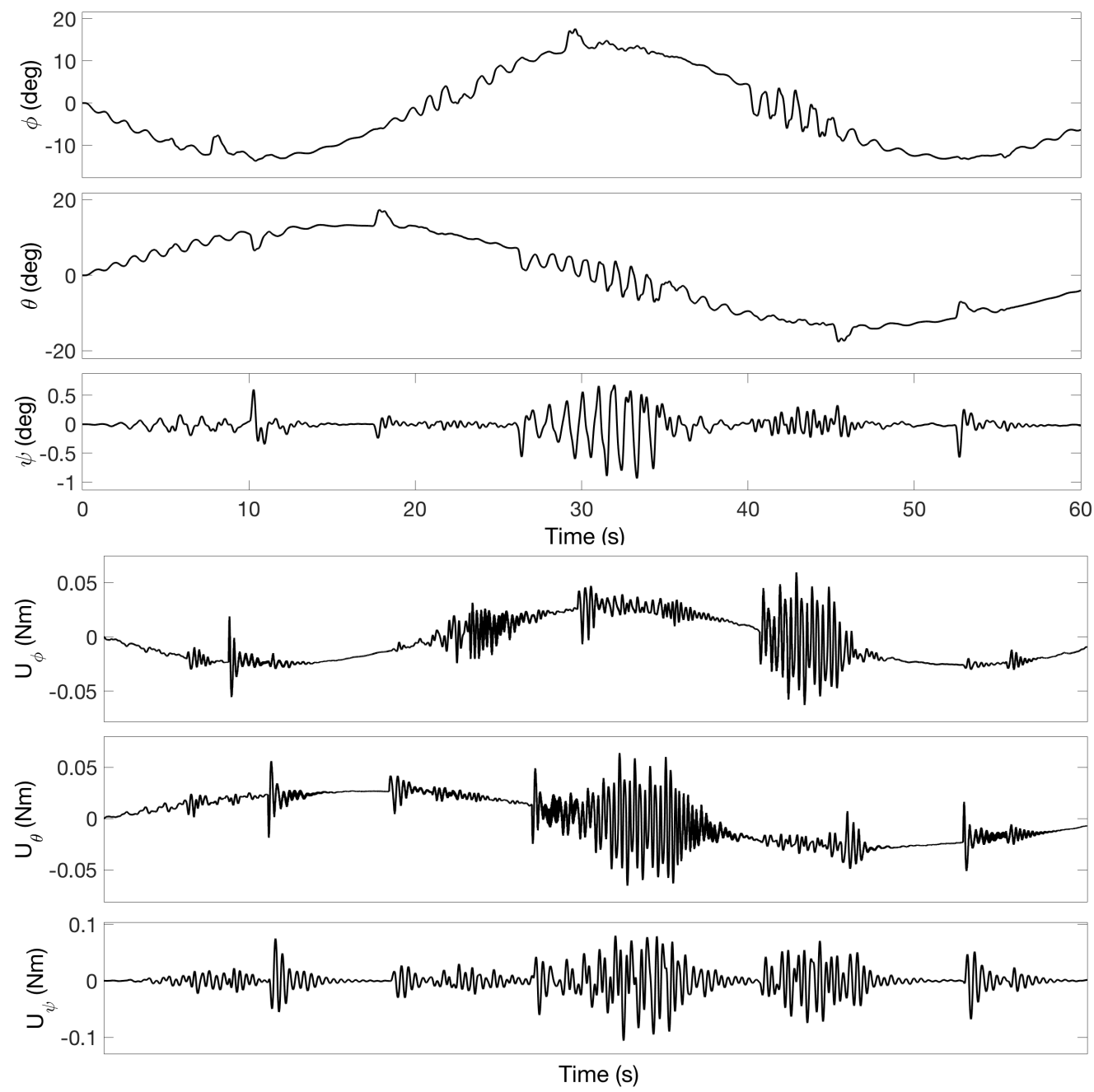

Figure 13: $\phi, \theta, \psi$ angles and respective controls for qc-SMC changing $\varrho_{i}$. 
functions are considered in eq. (17) which leads to the validity of the Theorem 4.5):

$$
u(t, X)=-\frac{\bar{h}(t, X)+\alpha}{\underline{g}(t, X)} \frac{[\dot{S}]^{2}+\beta S}{|\dot{S}|^{2}+\beta|S|},
$$

where $\alpha>0$ and $\beta>0$ are tuning parameters. In our case $\beta=1$ and $\underline{g}, \bar{h}$ are respectively the computed lower and the upper-bounds. Note that the control (A.3) is continuous everywhere outside of the origin.

Theorem 1. Consider the system in (A.1) and assume that the restrictions (A.2) are satisfied, then there exist $\alpha>0$ sufficiently big and $\beta>0$ such that the control (A.3) makes the system globally finite-time convergent.

\section{Acknowledgment}

The authors want to acknowledge Hauts-de-France region and ONERA to have financially supported this work.

\section{References}

[1] L. Planckaert, P. Coton, Quadrotor UAV aerodynamic model identification using indoor flight experiment and feasibility of UAV as wind gust sensor, in: International Micro Air Vehicles Conference and Flight Competition IMAV 2015, Aachen, Germany, 2015.

[2] E. Bernuau, D. Efimov, W. Perruquetti, A. Polyakov, On homogeneity and its application in sliding mode control, Journal of the Franklin Institute 351 (4) (2014) 1866-1901. doi:10.1016/j.jfranklin.2014.01.007.

[3] A. Polyakov, L. Fridman, Stability notions and Lyapunov functions for sliding mode control systems, Journal of the Franklin Institute 351 (4) (2014) 1831-1865. doi:10.1016/j.jfranklin.2014.01.002.

[4] R. Xu, U. Özgüner, Sliding mode control of a class of underactuated systems, Automatica 44 (1) (2008) 233-241. doi:10.1016/j.automatica.2007.05.014.

[5] M. Basin, L. Fridman, P. Shi, Optimal sliding mode algorithms for dynamic systems, Journal of the Franklin Institute 349 (4) (2012) 13171322. doi:10.1016/j.jfranklin.2012.02.013. 
[6] S. Bouabdallah, R. Siegwart, Backstepping and Sliding-mode Techniques Applied to an Indoor Micro Quadrotor, in: Proceedings of the 2005 IEEE International Conference on Robotics and Automation, 2005, pp. 2247-2252. doi:10.1109/ROBOT.2005.1570447.

[7] I. González, S. Salazar, R. Lozano, Chattering-Free Sliding Mode Altitude Control for a Quad-Rotor Aircraft: Real-Time Application, Journal of Intelligent \& Robotic Systems 73 (1-4) (2014) 137-155. doi:10.1007/s10846013-9913-8.

[8] B. Sumantri, N. Uchiyama, S. Sano, Least square based sliding mode control for a quad-rotor helicopter and energy saving by chattering reduction, Mechanical Systems and Signal Processing 66-67 (2016) 769784. doi:10.1016/j.ymssp.2015.05.013.

[9] E.-H. Zheng, J.-J. Xiong, J.-L. Luo, Second order sliding mode control for a quadrotor UAV, ISA Transactions 53 (4) (2014) 1350-1356. doi:10.1016/j.isatra.2014.03.010.

[10] H. Ramirez-Rodriguez, V. Parra-Vega, A. Sanchez-Orta, O. GarciaSalazar, Robust Backstepping Control Based on Integral Sliding Modes for Tracking of Quadrotors, Journal of Intelligent \& Robotic Systems 73 (1-4) (2014) 51-66. doi:10.1007/s10846-013-9909-4.

[11] C. L. Hwang, C. C. Chiang, Y. W. Yeh, Adaptive Fuzzy Hierarchical Sliding-Mode Control for the Trajectory Tracking of Uncertain Underactuated Nonlinear Dynamic Systems, IEEE Transactions on Fuzzy Systems 22 (2) (2014) 286-299. doi:10.1109/TFUZZ.2013.2253106.

[12] J.-J. Xiong, E.-H. Zheng, Position and attitude tracking control for a quadrotor UAV, ISA Transactions 53 (3) (2014) 725-731. doi:10.1016/j.isatra.2014.01.004.

[13] L. Besnard, Y. B. Shtessel, B. Landrum, Quadrotor vehicle control via sliding mode controller driven by sliding mode disturbance observer, Journal of the Franklin Institute 349 (2) (2012) 658-684. doi:10.1016/j.jfranklin.2011.06.031.

[14] A. Benallegue, A. Mokhtari, L. Fridman, High-order sliding-mode observer for a quadrotor UAV, International Journal of Robust and Nonlinear Control 18 (4-5) (2008) 427-440. doi:10.1002/rnc.1225. 
[15] R. Xu, U. Ozguner, Sliding Mode Control of a Quadrotor Helicopter, in: Proceedings of the 45th IEEE Conference on Decision and Control, 2006, pp. 4957-4962. doi:10.1109/CDC.2006.377588.

[16] L. Derafa, A. Benallegue, L. Fridman, Super twisting control algorithm for the attitude tracking of a four rotors UAV, Journal of the Franklin Institute 349 (2) (2012) 685-699. doi:10.1016/j.jfranklin.2011.10.011.

[17] G. Perozzi, D. Efimov, J.-M. Biannic, L. Planckaert, P. Coton, On sliding mode control design for UAV using realistic aerodynamic coefficients, in: 56th IEEE Conference on Decision and Control, Melbourne, Australia, 2017, pp. 5403-5408, dOI: 10.1109/CDC.2017.8264459.

[18] G. Perozzi, D. Efimov, J.-M. Biannic, L. Planckaert, P. Coton, Wind estimation algorithm for quadrotors using detailed aerodynamic coefficients, in: American Control Conference, Milwaukee, WI, USA, 2018.

[19] A. R. S. Bramwell, D. Balmford, G. Done, Bramwell's Helicopter Dynamics, Butterworth-Heinemann, 2001.

[20] W. Johnson, Helicopter Theory, Courier Corporation, 2012.

[21] G. J. Leishman, Principles of Helicopter Aerodynamics with CD Extra, Cambridge University Press, 2006.

[22] S. Bouabdallah, R. Siegwart, Full control of a quadrotor, in: 2007 IEEE/RSJ International Conference on Intelligent Robots and Systems, 2007, pp. 153-158, dOI: 10.1109/IROS.2007.4399042. doi:10.1109/IROS.2007.4399042.

[23] H. K. Khalil, Nonlinear Systems, Prentice Hall, 2002.

[24] S. Ding, A. Levant, S. Li, Simple homogeneous sliding-mode controller, Automatica 67 (2016) 22-32. doi:10.1016/j.automatica.2016.01.017.

[25] W. Perruquetti, T. Floquet, E. Moulay, Finite-Time Observers: Application to Secure Communication, IEEE Transactions on Automatic Control 53 (1) (2008) 356-360. doi:10.1109/TAC.2007.914264. 